\title{
Dendrimers in Cancer Treatment and Diagnosis
}

\author{
Srinivasa-Gopalan Sampathkumar, and Kevin J. Yarema
}

1.1

Overview

Dendrimers are nano-sized, radially symmetric molecules with well-defined, homogeneous and monodisperse structure consisting of tree-like arms or branches. Over the past two decades since the term "dendrimer" was formally defined, research interest in these molecules has gradually evolved from a primary focus on overcoming purely synthetic challenges to include aesthetic and theoretical perspectives, and, more recently, with the ongoing flurry of "nanobiotechnology" advances, to develop practical and commercial applications for these elegant nanodevices. Today, a critical mass of knowledge exists to synthetically control the physicochemical properties of dendrimers and thereby govern their ensuing biological behaviors. These fundamental scientific advances, coupled with practical methods to covalently conjugate a wide range of bioactive molecules to the surface of a dendrimer or encapsulate them as guest molecules within void spaces, provide a highly versatile and potentially extremely powerful technological platform for drug delivery. This chapter recaps synthetic advances in dendrimer construction and summarizes the many features of these fascinating macromolecules that endow them with favorable properties for drug delivery applications. Finally, with this enticing technology having matured to the point where it is ready to confront "real-world" challenges, a synopsis is outlined of the prospects for exploiting dendrimer-based nanodevices for one of the most intractable medical challenges, the diagnosis and treatment of cancer.

1.2

Introduction

The discovery, design, and development of anticancer therapeutic agents have proven to be remarkably intractable despite intense efforts at the research and clinical levels over many decades. A brief consideration of the challenges facing an

Nanotechnologies for the Life Sciences Vol. 7

Nanomaterials for Cancer Diagnosis. Edited by Challa S. S. R. Kumar

Copyright (@) 2007 WILEY-VCH Verlag GmbH \& Co. KGaA, Weinheim

ISBN: 978-3-527-31387-7 
$2 \mid 1$ Dendrimers in Cancer Treatment and Diagnosis

anticancer drug illustrates some of the reasons for frustratingly-slow progress: first the drug must be able to seek out subtle changes that distinguish a transformed cell from the other 200 or so types of healthy cells found in the body and then provide a sufficiently high dose of a toxic agent to kill the cell. The difficulty of this task is amplified by the potential metastasis of cancer cells to widely-spread niches throughout the body, each with unique properties. Furthermore, to successfully cure a patient, each and every cancer cell must be eradicated because even one in a thousand - often harboring latent resistance - can re-grow into a second tumor refractory to therapeutic intervention.

Readers of this chapter, contained within a volume devoted to the development of novel cancer therapeutics, do not require convincing of the difficulty of combating cancer and this issue will not be labored here. Instead, this chapter provides a broad overview of dendrimer-based nanotechnologies for the treatment of cancer with a consideration of their synthesis, the encapsulation and covalent attachment of drugs, and various strategies used for tumor specific targeting, imaging, and therapy. The discussion of specific topics begins with a description of the basic properties of dendrimers in Section 1.3 to highlight how these molecules lie at the interface between conventional synthetic polymers and the archetypical nanosized biological polymers, proteins. Section 1.4 briefly outlines the synthesis of dendrimers; exhaustive review articles (referenced therein) provide a wealth of synthetic detail beyond the scope of this discussion. This chapter aims to provide the reader with the knowledge that, by control of design parameters, the attributes of dendrimers can be tuned to incorporate the most desired features of synthetic polymers and proteins and, thereby, gain exquisite control of biological activity.

Upon having established that dendrimers are synthetically-tractable, biologicallycompatible nano-devices, their unique suitability for drug delivery will be delineated in some detail in Section 1.5. Specific topics covered include the alternative drug-carrying strategies of encapsulation (Section 1.5.2) and covalent conjugation (Section 1.5.3), as well as design features needed to ensure bioactivity of the drug (Section 1.5.4) and the biocompatibility of the dendrimer (Section 1.5.5). Finally, with the multi-disciplinary set of tools required for dendrimer-based drug delivery now reaching maturity, this area of investigation is undergoing transformation from the developmental stage to "real-world" applications. Accordingly, Section 1.6 discusses the prospects for using dendrimer-based nanotechnologies to overcome arguably the most difficult biomedical problem now faced, the diagnosis and treatment of cancer. In particular, the general properties of dendrimers that make them attractive for cancer treatment will be outlined in Section 1.6.1, with a specific benefit - exploitation of the enhanced permeability and retention effect that allows passive accumulation at the sites of tumors - discussed in Section 1.6.2. The ability of dendrimers to serve as a technological platform for multifunctional nano-devices that include targeting, imaging, and/or cytotoxic modalities is discussed in Section 1.6.3 and their prospects for diagnosis and therapeutic applications are given in Sections 1.6.4 and 1.6.5, respectively. Finally, Section 1.6.6 gives a brief synopsis of innovations that promise to speed progress in the near future. 
Together - while broader in scope than the typical discussion of dendrimers, drug delivery, or cancer therapy - this chapter provides an integrated look at the many considerations required for successful application of dendrimers for cancer therapy. For a more-in-depth consideration of any particular sub-topic, the interested reader is urged to consult the many original research reports and review articles cited throughout.

\section{3}

Basic Properties and Applications of Dendrimers

1.3.1

Structural Features and Chemical and Biological Properties

\subsubsection{Basic Features of Dendritic Macromolecules are Inspired by Nature}

Dendritic structures, characterized by hyperbranched subunits, are widely found in nature. Indeed, the word dendrimer is based on the Greek words "dendron" meaning "tree" or "branch" and "meros" meaning "part" $[1,2]$. Taken literally, similarities with dendrimer macromolecules are illustrated by a tree, where the leaves of a tree are maximally displayed on a highly-branched scaffold to maximize their accessibility to the outside world to optimize functions such as light harvesting. The branches of a tree can modify the environment within them, similarly the core/ interior encapsulated within a dendrimer can provide a sheltered microenvironment with tailored chemical properties and reactivities [2]. In addition to actual trees, Nature has scaled dendrimeric structures down to the multi-centimeter level (the intricate neural pathways found in the brain), the millimeter level (ice crystals and snowflakes), and yet further to the micron level (the dendritic outgrowths of neurons). At a molecular, "nano-size" level, dendrimer-like molecules, such as branching polysaccharides, provide an elegant solution to a cell's need to stably store high energy molecules like monosaccharides; the presence of many chain ends allows the rapid release of large numbers of glucose monomers when needed [3].

Unlike Nature, which provides dendritic structures in a range of sizes from real trees to the namesake molecular nano-sized structures, this chapter focuses exclusively on dendritic macromolecules that are of a synthetically tractable scale and appropriate for cancer therapy. Starburst ${ }^{\circledR}$ clusters [4], made of poly(amidoamine) (PAMAM) units, are arguably the most-thoroughly characterized and extensivelyutilized dendrimers [5]. A basic characteristic of these molecules is their layered composition - known as "cascades" or "generations" [1] (Fig. 1.1). The overall shapes of dendrimers range from spheres to flattened spheroids (disks) to amoeba-like structures, especially in cases where surface charges exist and give the macromolecule a "starfish"-like shape [6].

The exact morphology of a dendrimer depends both on its chemical composition (the chemical composition of PAMAM dendrimers is shown in Fig. 1.1) as well as on the generation number, as exemplified by PAMAM where the lowest generation 


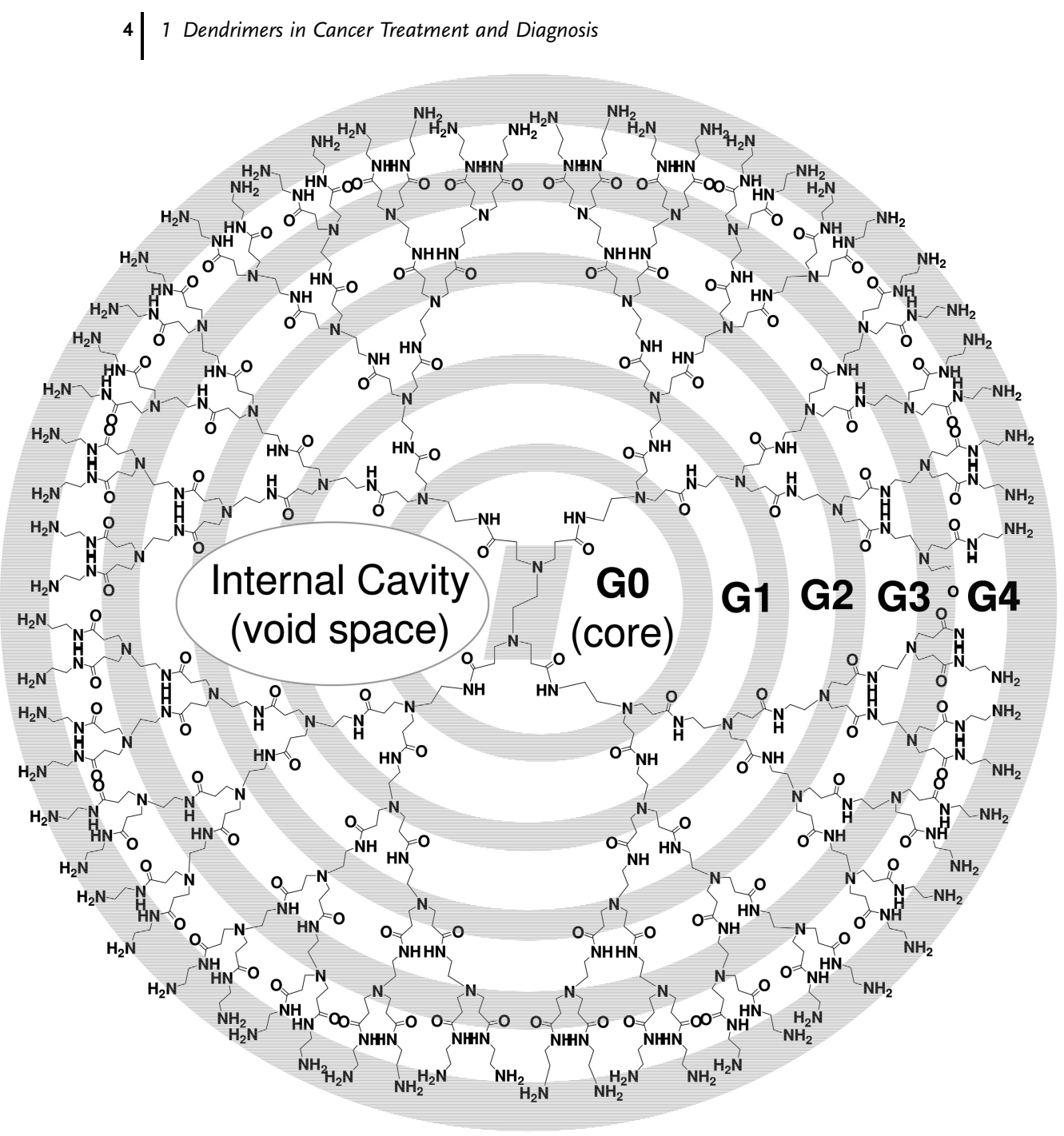

Fig. 1.1. Schematic representation of a generation $\mathrm{G} 4$ dendrimer with 64 amino generations. The periphery of successive generations is marked by grey circles, starting groups at the periphery. This dendrimer starts from an ethylene diamine core; the branches or arms were attached by exhaustive Michael addition to methyl acrylate followed by exhaustive aminolysis of the resulting methyl ester using ethylene diamine [36]. This sequence of reactions was applied in an iterative fashion to increase the level of from $G 0, G 1, G 2, G 3$ and G4. Of note,

distinctive features of dendrimers, including the densely-packed membrane-like arrangement of surface functional groups, the formation of internal cavities, and the condensation into globular structures (not shown), are typically manifest at the $\mathrm{G} 4$ stage (and amplified in successive generations, Table 1.1). 
structure (e.g., G0 and G1) have highly asymmetric shapes and posses open structures compared with higher-generation structures that first appear to be disk-like and then progress to increasingly spherical geometries [5] as they assume globular structures with a significant reduction in hydrodynamic volume [7]. In addition to sphere-like dendrimers - based on a "dot-like" core (Fig. 1.1) - increasing interest is developing in cylindrical dendrimers that are based on "rod-like" cores. These interesting spin-off macromolecules have been compared with spaghetti because they can be rigid like the uncooked form of this pasta or highly flexible like the cooked form; these properties can be tuned based on the chemical composition and density of packing of the dendritic branches [6]. Additional features of dendrimers are discussed below, by comparison with the two classes of molecules they are most often likened to, i.e., "conventional" synthetic polymers and, the most extensively studied biological polymer, proteins.

\subsubsection{Comparison of the Properties of Dendrimers and Conventional Synthetic Polymers}

Dendrimers have both similarities and differences when compared with traditional polymers. One similarity is the vast diversity in the basic monomeric building blocks used to create both classes of molecules and to provide the final macromolecular products with a wide range of chemical, mechanical, and biological properties. Until recently, polymer chemistry has been focused on the production of linear polymers that often have a degree of branching or crosslinking; this property, however, is dramatically limited by comparison to dendrimers whose entire identity is wrapped up in their hyperbranched character. Interestingly, highly-branched polymers of the same material can be vastly different from conventional polymers of a similar molecular weight and composition; in particular, as dendritic macromolecules progress in size, usually when becoming larger than the third generation (G3), they assume globular structures and occupy considerably smaller hydrodynamic volumes than linear polymers [1].

When dendrimers condense into globular structures, a feat rarely achieved with linear synthetic polymers, their many termini become fixed into an outwards orientation and also form a densely packed, membrane-like surface (Fig. 1.1). This structural arrangement provides numerous attachment points for covalent conjugation of bioactive molecules on the surface as well as enclosed cavities for occlusion of guest molecules within the dendrimer. This tight packing ultimately results in the reaching of a critical branched state - known as the "starburst effect" [4] where growth of the dendritic macromolecule is dramatically hindered by steric constraints [8] (this state is reached at G10 or G11 for PAMAM, Table 1.1). Dendrimers also have dramatically different rheological properties than conventional polymers; viscosity tends to increase continuously with molecular mass for linear macromolecules whereas the intrinsic viscosity of dendrimers goes through a maximum at approximately the fourth generation and then declines $[8,9]$. Finally, dendrimers have a negligible degree of polydispersity because, unlike classical polymerization that is random in nature and produces molecules of various sizes, the size of dendrimers can be carefully controlled during synthesis. Under ideal condi- 
6| 1 Dendrimers in Cancer Treatment and Diagnosis

Tab. 1.1. Generation by generation specifications for PAMAM

Starburst ${ }^{\circledR}$ dendrimers. (Adapted from Ref. [5].)

\begin{tabular}{|c|c|c|c|c|}
\hline \multirow[t]{2}{*}{ Generation } & \multicolumn{4}{|c|}{ Physical or structural parameter } \\
\hline & $\begin{array}{l}\text { Molecular weight } \\
\text { (Daltons) }\end{array}$ & $\begin{array}{l}\text { Diameter } \\
(\AA)\end{array}$ & $\begin{array}{l}\text { Surface groups } \\
\left(-\mathrm{NH}_{2}\right)\end{array}$ & $\begin{array}{l}\text { Radius of } \\
\text { gyration }(\AA)\end{array}$ \\
\hline G0 & 517 & 15 & 4 & 4.93 \\
\hline G1 & 1430 & 22 & 8 & 7.46 \\
\hline G2 & 3256 & 29 & 16 & 9.17 \\
\hline G3 & 6909 & 36 & 32 & 11.2 \\
\hline G4 & 14215 & 45 & 64 & 14.5 \\
\hline G5 & 28826 & 54 & 128 & 18.3 \\
\hline G6 & 58048 & 67 & 256 & 22.4 \\
\hline G7 & 116493 & 81 & 512 & 29.1 \\
\hline G8 & 233383 & 97 & 1024 & 36.4 \\
\hline G9 & 467162 & 114 & 2048 & 46.0 \\
\hline G10 & 934720 & 135 & 4096 & 55.2 \\
\hline G11 & 1869780 & 167 & 8192 & 68.3 \\
\hline
\end{tabular}

tions, preparations of dendrimers are monodisperse, which is to say they have one molecular weight instead of the range, over tens or even hundreds of $\mathrm{kDa}$, often seen for traditional synthetic polymers. Indeed, the homogeneity and uniformity of dendrimers of successive generations becomes strikingly obvious as shown by the tunneling electron microscopy (TEM) images for G5 to G10 PAMAM (Fig. 1.2) $[10]$.

\subsubsection{Comparison of the Properties of Dendrimers and Proteins (a Biological Polymer)}

As discussed above, dendrimers have unusual, often dramatically different, characteristics compared with conventional synthetic polymers. In fact dendritic molecules have often been compared with proteins, which are the workhorse biological polymers. Both classes of macromolecules are globular, are composed of precisely controlled monomeric units, have defined architectures, are of comparable size (Table 1.1), and have surfaces with chemically-reactive sites that can be endowed with biologically-compatible ligands found on proteins (such as glycosylation, Section 1.4.3.2). Moreover, the interior of a dendritic molecule, reminiscent of a protein, can harbor unique microenvironments, providing behaviors like redox chemistry, molecular recognition, ligand and substrate binding, and catalysis $[11,12]$. The ability to create and exploit isolated nanoenvironments within a dendritic shell is derived from two main properties of a dendrimer. First, dendritic macromolecules adopt a semi-globular or fully globular character containing internal void 


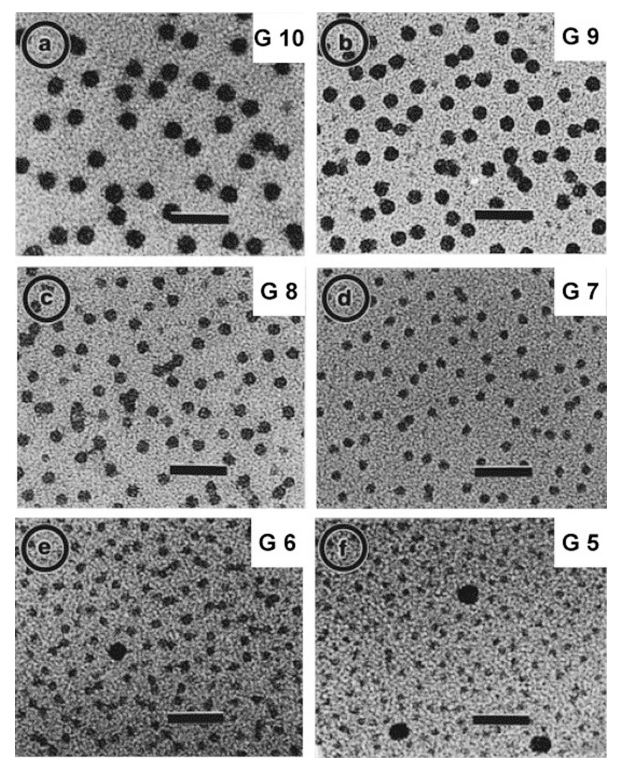

Fig. 1.2. Transmission electron microscopy (TEM) of PAMAM dendrimers. Dendrimers were positively stained with aqueous sodium phosphotungstate and imaged by conventional TEM: (a) G10, (b) G9, (c) G8, (d) G7, (e) G6, (f) G5. The scale bars indicate $50 \mathrm{~nm}$, and a

small amount of G10 has been added as a focusing aid for G6 and G5. (Reprinted with permission from Jackson and coauthors [10]. Copyright 1998 by the American Chemical Society.)

spaces once they reach the fourth generation in size (Fig. 1.1) [8], enabling the encapsulation of protein-like functions, including catalysis [13, 14]. Second, these molecules have molecular flexibility and can undergo deformations, leading to rudimentary "lock and key" molecular recognition of the type vitally important to protein functions $[15,16]$.

Molecular recognition between molecules is a fundamental process in biology and chemistry without which life could not exist. The concept of molecular recognition, based on complementarity between the receptors and substrates, is very similar to the "lock and key" function first described by Emil Fischer over 100 years ago. In biology, the "lock" is the molecular receptor such as a protein or enzyme and the "key" can be regarded as a substrate such as a drug or ligand that is recognized to give a defined receptor-substrate complex [15]. In proteins, molecular recognition is largely driven by non-covalent forces such as hydrogen bonding, electrostatics, van der Waals forces, $\pi-\pi$ interactions, solvent-dependent interactions including hydration forces, and conformational energy [17]; notably, all of these parameters can be controlled in dendrimers through synthetic design. The inherent ability of dendrimers to achieve molecular recognition of biological features, if it can be successfully developed to a level of sophistication where it can be exploited for the recognition of the surface biomarkers that distinguish cancer 
$8 \mid 1$ Dendrimers in Cancer Treatment and Diagnosis

cells from healthy cells (Section 1.6.3), has important - and extremely beneficial implications for drug delivery (Section 1.5).

Although sharing many superficial features, a close inspection reveals important differences between dendrimers and proteins. For example, remaining on the topic of deformability and flexibility, the linear, folded chain of a protein is more tightly packed but also has a greater potential for flexibility (when a comparison is made between the fully folded and unfolded states of a peptide chain) than is possible for the branches of a dendrimer. Only a small proportion of the potential flexibility of a protein, however, is usually available for "induced fit" interactions because the extensive unfolding of a protein is highly thermodynamically unfavorable. By comparison, although the extensive covalent bond networks within a dendrimer prevents complete unfolding under any condition, this arrangement does provide sufficient flexibility to allow dramatic - albeit somewhat thermodynamically unfavorable - deformations fairly readily [18]. Next, to consider dendrimer surfaces in comparison with proteins, synthetic dendritic macromolecules can be given a significant repertoire of tunable characteristics not found on natural proteins; this feature has greatly propelled the development of practical applications for these molecules. In particular, the surface of a protein contains a relative sparse complement of chemically reactive and accessible functional groups because most amino acid side chains are buried with the globular structure of the protein. By contrast, virtually all of the termini of dendritic branches, which can be customized with a wide range of chemical functionalities (Fig. 1.3), are oriented outward and are highly accessible on the surface of the dendrimer (Fig. 1.1). The consequent ability

of a dendrimer to be functionalized with far more surface groups than a protein of comparable size $[1,19]$ has provided impetus to their widespread use as drug delivery vehicles.

\section{3 .2}

\section{Dendritic Macromolecules Possess a Wealth of Possible Applications}

Within the past decade, the success of chemists in synthesizing mimics of natural dendrimers with a plethora of interesting physicochemical properties at the nanoscale has spurred efforts to find practical uses for these versatile nanodevices. Now that efforts to synthesize these molecules have reached fruition, there is a pleasing circularity that certain applications mirror natural processes considering that dendritic molecules were initially inspired by nature. In a dramatic example, a primary function of the leaves of real trees is for light harvesting; now, synthetic dendrimers have been created with highly-efficient light-harvesting antennae as well $[8,20]$. Similarly, the dendritic network of hairs found on the Gecko foot that allows amazingly strong attachment to many types of surfaces through van der Walls forces [21] has led to efforts to create new forms of adhesives that are unaffected by the roughness, smoothness, wetness, or other macroscopic properties of the surface while providing strong but reversible adhesion. In addition to these two examples, many novel applications such as the exploitation of organometallic dendrimers as quantum dots for imaging, the solubilization of hydrophilic dyes in 

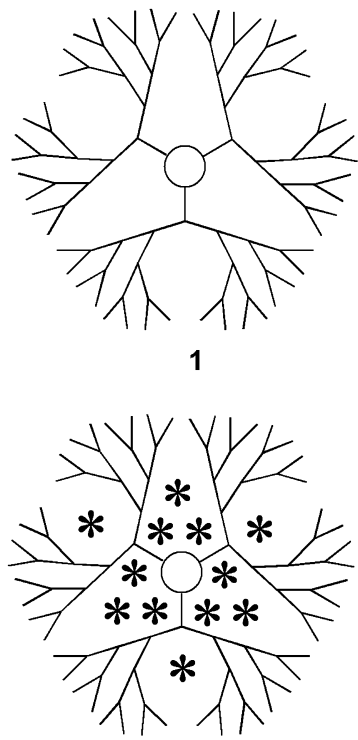

4

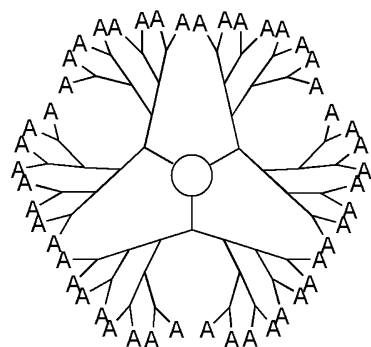

7

Fig. 1.3. Structural options for dendrimerbased drug delivery. Dendrimers can be synthesized with neutral surfaces (1) and positive (2) or negative (3) charges at the periphery; moreover, dendritic macromolecules, generally when larger than G3, can harbor non-covalently encapsulated guest/drug molecules [ 4 and discussion in Section 1.5.2]. An alternative strategy for drug delivery is through covalent conjugation of ligands (" $A$ " in 5) to the surface of the dendrimer (Section 1.5.3). The versatility of dendrimers for drug delivery is illustrated by considering that " $A$ " could be a targeting

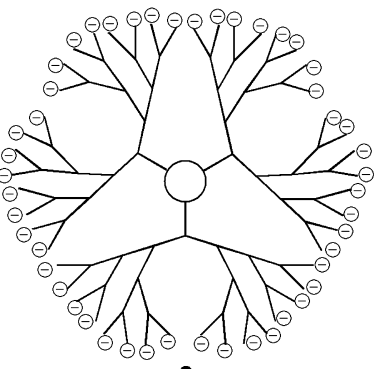

3

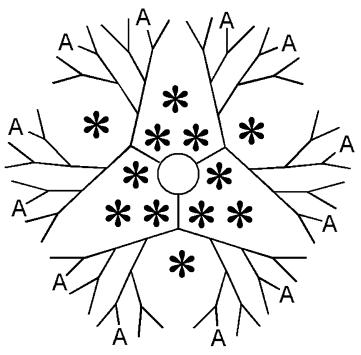

6

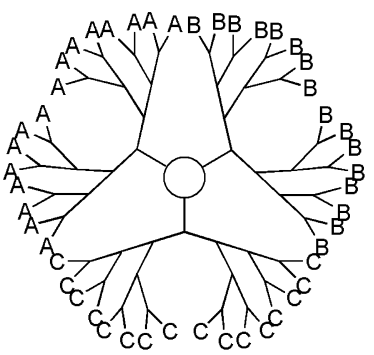

9 ligand (Section 1.6.6.3) and the active drug could be encapsulated within the same macromolecule (6). Synthetic strategies are now available for providing dendritic clusters with extremely high densities of surface ligands (7) and for providing more than one type of surface ligand, either in a random orientation (8), or in blocks (9). The latter dendrimers are now being exploited in sophisticated cancer cell targeting (Fig. 1.4) and drug release (Section 1.5.3.3) strategies where A, B, and C can be any combination of targeting agents, drugs, contrast agents, or functional groups that improve pharmacological properties. 
apolar dendritic "solvents" [22], use as chemical catalysts, and in electronics as insulated molecular wires, light-emitting diodes, or fiber optics [12, 23, 24] have been reported. Besides their use for drug delivery and cancer therapy, the many emerging chemical, synthetic, research, and industrial uses for dendrimers are outside the scope of this article and will not be discussed further; the interested reader can consult chapter articles $[1,2,11]$.

\section{4}

Methods for Dendrimer Synthesis

1.4.1

History and Basic Strategies

The ability to create homogeneous molecules with defined dendritic architecture and novel physicochemical properties at the sub-nano to nano-size scales occurred in chronological synchrony with the wide-spread application of nanobiotechnology to biology and medicine. Consequently, the parallel development of synthetic chemical methodology and the ever-increasing application of nano-tools in biomedicine triggered an explosive growth in the new field of dendrimer synthesis. This growth is evidenced by a cursory search for "dendrimers and synthesis" in the Web of Science database, which reveals that 2000 articles have been published on this topic since 1986. Clearly, a full discussion is beyond the scope of this report; excellent accounts and review articles on the synthesis of dendrimers by pioneers of the field have appeared at regular intervals [25-32] and are cited throughout this chapter. Nonetheless, a working knowledge of the chemical properties of dendrimers is critical to successfully devise efficacious therapeutic strategies with these versatile, but temperamental, macromolecules (as described in detail in Sections 1.5 and 1.6). Accordingly, we next provide an outline of the basic strategies and building blocks employed in dendrimer synthesis, with an emphasis on families of dendritic molecules that possess special properties - such as possessing cavities in their interiors suitable for host-guest complexation similar to enzymesubstrate complexes or displaying several functional groups on their surface appropriate for sophisticated drug delivery strategies - relevant to the field of biology and medicine.

\subsubsection{Cascade Reactions are the Foundation of Dendrimer Synthesis}

Although the term "dendrimer" was coined by Tomalia and coworkers less than two decades ago, the basic cascade or iterative methods that are currently employed for synthesis were known to chemists much earlier. For example, similar schemes form the basis of solid phase peptide synthesis. In turn, biology has long exploited similar iterative strategies in biochemical synthetic pathways; one example is provided by fatty acid biosynthesis [33]. Focusing on dendrimers, these macromolecules are constructed by performing simple chemical reactions in a repetitive or iterative manner by using small building blocks. In 1978 Vögtle and coworkers re- 
A. Cascade synthesis of polyaza compounds by Vögtle and coworkers (1978)

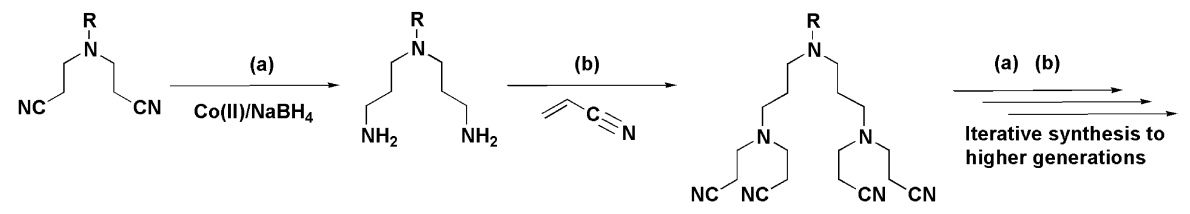

B. Divergent synthesis of poly(amidoamine) dendrimers (PAMAM) by Tomalia and coworkers (1986)
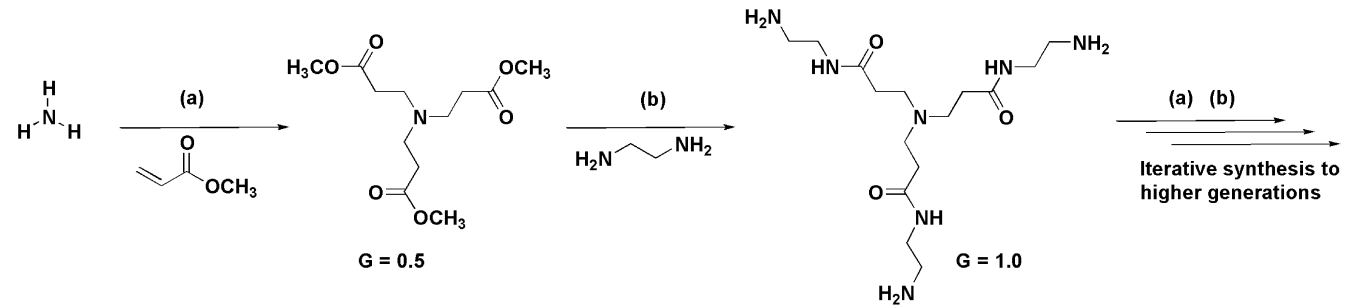

C. Convergent synthesis of aryl ether dendrimers by Fréchet and coworkers (1990)

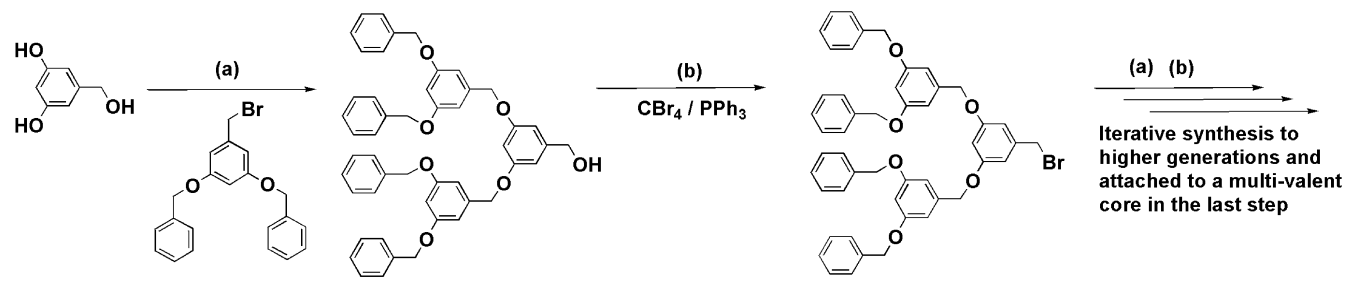

Fig. 1.4. Synthetic approaches to dendrimers. (A) Cascade reaction sequences developed for the synthesis of "non-skid-chain like" polyaza macrocyclic compounds [34]. (B) Divergent approach - synthesis of radially symmetric PAMAM dendrimers using ammonia as the trivalent core; the generations are added at each synthetic cycle (two steps), leading to an exponential increase in the number of surface functional groups [36]. (C) Convergent approach - synthesis of dendrons or wedges or branches that will become the periphery of the dendrimer when coupled to a multivalent core in the last step of the synthesis [40].

ported a similar approach, termed as cascade reactions, for the construction of nonskid-chain-like poly-aza macrocyclic molecules with well-defined architectures. Cascade synthesis is defined as "reactions where a functional group (e.g., amine) is made to react in such a way as to appear twice in the subsequent molecule or product" [34] (Fig. 1.4A). In the first step of the synthesis a primary or secondary amine was reacted with excess acrylonitrile in a Michael reaction to obtain a product with two arms [bis(2-cyanoethyl)amines]. In the second step the nitrile groups were reduced using cobalt(II)/sodium borohydride to generate a new set of primary amine groups on both arms. The newly generated amino groups were then subject to identical reaction sequences iteratively to obtain the desired oligo-amine compounds. 
In 1985 Newkome and coworkers reported the synthesis of cascade molecules consisting of hydrocarbon core and shell with alcohol groups on the surface. These synthetic efforts were inspired by the Leuwenberg model of arboreal design; hence they named their synthetic macromolecular tree-like molecules "arborols" (Latin: arbor $=$ tree). Interestingly, characterization of these molecules showed they could be considered to be unimolecular micelles possessing cavities for encapsulation [35], a property that foreshadowed today's efforts to use dendrimers for the delivery of encapsulated small molecule drug candidates (Section 1.5.2).

\subsubsection{Dendrimer Synthesis has Expanded Dramatically in the Past Two Decades}

In 1986, Tomalia and coworkers coined the now popular name "dendrimers" (Greek: dendron = branch or tree-like) for radially symmetric branched molecules and reported the application of cascade synthesis for the synthesis of starburst dendrimers [36]. These researchers obtained homogeneous dendrimers by using a synthetic sequence of two simple reactions: (a) exhaustive Michael addition of ammonia to methyl acrylate and (b) exhaustive aminolysis of the resulting tri-ester derivative by ethylene diamine. The acrylate addition and aminolysis were repeated in an iterative manner, with excellent yields in each step, to prepare various molecules with increasing molecular weight and generations (Fig. 1.4B). The products with ester groups at the exterior were defined as $\mathrm{G}(m+0.5)$ generations and those with amine groups at the exterior were defined as $\mathrm{G}(m)$ (Fig. 1.1). This simple methodology is both powerful and versatile as it provided the ability to synthesize dendrimers with various surface properties. For instance, the ester groups could be hydrolyzed to present negatively charged carboxylate functional groups at the periphery or the amine groups could be protonated to present positive charges at the periphery. Electron micrographic studies showed the dendrimer with carboxylate groups of generation, $\mathrm{G}=4.5$, to be highly monodispersed with a diameter of $88 \pm 10 \AA$, compared with the theoretical value of $\sim 78 \AA$. These dendrimers, when covalently attached to a polymeric backbone, were called "starburst polymers" or, less commonly, "cauliflower polymers" [7, 28].

1.4 .2

Strategies, Cores, and Building Blocks for Dendritic Macromolecules

\subsubsection{Dendrimers are Constructed from Simple "Building Blocks"}

In terms of synthesis, dendrimers can be constructed by using simple chemical reactions and building blocks reminiscent to the modular assembly of "LEGO" toys. Due to the ease, simplicity and repetitive nature of the synthetic methods, dendrimers based on organic, inorganic and organometallic molecular building blocks with greater than hundred different compositions are currently known, and new designs continue to be reported at a fast pace. In general, dendrimers consists of three major regions - (a) an initiator core, (b) a shell with extending arms or branches made of building blocks and (c) the exterior or outer-most surface groups on the termini of the branches. 
There are innumerable ways of designing dendrimers [37-39]. For instance, the symmetry of the initiator core (Fig. 1.1) can be varied by using a wide range of molecules, which have included ammonia, $\alpha, \omega$-diaminoalkanes, tri-substituted benzene, oligo- or polyalcohols, nucleic acids, amino acids, lipids, carbohydrates, or heteroatoms; many additional permutations are possible, e.g., the number of branching units in the initiator can be increased (tri- or tetra- or higher valency cores have been reported). Once the core moiety has been selected, options for the synthesis of the dendritic branches are equally numerous as various types of building blocks can be used, either singly or in combination with each other in the same dendritic macromolecule. The lengths of the dendritic arms, the nature of the surface, and the display of terminal functional groups can all be customized.

\subsubsection{The Synthesis of Dendrimers Follows Either a Divergent or Convergent Approach}

Dendrimers can be synthesized by two major approaches. In the divergent approach, used in early periods, the synthesis starts from the core of the dendrimer to which the arms are attached by adding building blocks in an exhaustive and step-wise manner. This process provides dendrimers with incrementally increasing generation numbers. However, only one type of reaction can be performed at each step, giving a uniform display of only one functional group on the exterior surface; moreover, defects in successive generations can arise due to incomplete reactions or steric hindrance (Fig. 1.4B).

In the convergent approach, pioneered by Fréchet and coworkers [40], synthesis starts from the exterior, beginning with the molecular structure that ultimately becomes the outer-most arm of the final dendrimer (Fig. 1.4C). In this strategy, the final generation number is pre-determined, necessitating the synthesis of branches of the various requisite sizes beforehand for each generation [31]. Small branches or dendrons are synthesized starting from the building blocks containing surface groups; these assemblies are then condensed with a multivalent core. This approach is versatile in the sense that branches of different molecular composition can be linked to a single core molecule, introducing regional variations on the final dendrimer (Fig. 1.3); this strategy also minimizes the introduction of defects at various stages of synthesis.

\section{4 .3}

\section{Heterogeneously-functionalized Dendrimers}

\subsubsection{Basic Description and Synthetic Considerations}

By simultaneously conjugating appropriate targeting moieties, drugs, and imaging agents to dendritic polymers, "smart" drug-delivery nanodevices can be developed to target, deliver, and monitor the progression of therapy. For example, as will described in greater detail below, a dendrimer intended for cancer therapy needs to be functionalized with the drug itself, display a moiety for targeting to the tumor cells, as well as include surface groups designed to improve the pharmacological 
properties (e.g., to ensure water solubility, avoid non-specific uptake or immunogenicity). Several synthetic strategies - primarily the convergent method discussed above (Section 1.4.2.2) - have been developed that enable multiple species to be added to a dendritic surface in an ordered manner [41] and thereby achieve multiple functionalities within the same dendritic nanodevice (Fig. 1.3). The ability to create multi-functional nanodevices based on dendritic scaffolds, however, remains a challenging endeavor because conjugating several types of different molecules to a dendrimer is likely to change its physicochemical properties and resulting biological activity. Practically, additional synthetic steps required to fine-tune bioactivity and remedy bioincompatibility if it arises may render the whole process costinefficient at best and, more troublesomely, lead to loss of product uniformity, thereby negating a key benefit of dendrimers, i.e., their monodisperse, fully defined nature [42].

\subsubsection{Glycosylation is an Example of Surface Modification with Multiple Bioactivities}

An outstanding demonstration of the synthetic power of decorating the surface of dendrimers with "interesting" molecules comes, once again, by way of comparison of these nanodevices with proteins. Proteins, which have had the opportunity to evolve biocompatibility and systemic functions in multicellular organisms over hundreds of millions of years, have found it advantageous to decorate their surfaces with complex carbohydrates when they are displayed on the cell surface or secreted into the extracellular milieu. In the past few years, it has become clear that these sugars play many key roles in molecular recognition over short distances, such as interactions with the extracellular matrix and with neighboring cells, as well providing system-wide communication (e.g., almost all protein hormones are glycosylated). When developing dendritic nanotools requiring bioactivity similar to that found in proteins, including the ability needed by a drug candidate to seek out and evoke responses at a specific but far-removed cell type in the body, it is wise to learn from nature and consider the inclusion of sugars to be an important design parameter.

The ability to provide dendrimers with oligosaccharide coatings has been facilitated by the many functional groups that can be displayed on the surface and function as chemical handles for covalent attachment of a second group. A pioneering example of sugar display on a dendritic scaffold was provided by the unusual ninecarbon sugar sialic acid [43, 44]. This sugar, when displayed on human cells, serves as a critical binding epitope for the influenza virus. The virus, however, does not bind to soluble sialic acid, or sialic acid appended to a conventional polymer. Because these forms of sialic acid do not serve as effective binding elements, they are unable to act as a molecular decoys [45] and prevent the virus from binding to its real target, sialic acid on the human cell. By contrast, when sialic acid was conjugated to the surface of a dendritic polymer, it functioned as an effective and efficient binding decoy [46, 47], opening the door to the development of new diagnostic devices and novel anti-viral therapies [48, 49]. The molecular basis of the preferential recognition of sialic acid by the influenza virus when this sugar was 
displayed on a highly structured dendritic scaffold was traced to the "cluster glycoside effect" [50]. Over the past decade it has become firmly established that carbohydrate-based recognition depends on multiple simultaneous interactions to increase specificity and affinity $[45,51]$. The demonstration that dendrimers provide an ideal synthetic platform for the appropriate display of carbohydrates to achieve the cluster glycoside response [52-54], along with improved methodology to synthesize glycoconjugated-dendrimers [43, 55], has driven the expansion of this approach from a single monosaccharide to a sugar-amino acid couple (the Tn antigen, which is $N$-acetylgalactosamine linked to serine [56]) to disaccharides (lactose [57] and the T-antigen [58]), and, finally, to tetrasaccharides (the sialyl Lewis X epitope [59]).

1.5

\section{Dendrimers in Drug Delivery}

1.5.1

Dendrimers are Versatile Nano-devices for the Delivery of Diverse Classes of Drugs

A successful drug must perform the demanding tasks of selectively recognizing and binding to a molecular target, then triggering an appropriate biological response, all the while possessing pharmacological properties that render it "drug-like". In some cases, nature has supplied compounds - such as aspirin or penicillin - that can be used directly as drugs but the more common situation is that many otherwise promising therapeutic agents are not successful in the clinic because of their poor pharmacological properties. The properties of dendrimers, in particular the synthetic ability to provide them with many different biological properties, along with their capacity to carry conjugated surface molecules or encapsulated guest molecules, make them immediately attractive as potential vehicles for drug delivery.

Drug delivery efforts are complicated by the diversity of molecules that hold potential therapeutic or diagnostic value; briefly reviewing three classes of drug candidates based on size demonstrates the wide applicability of dendrimers to drug delivery. First, regarding "small molecules", many low molecular weight drug candidates are limited by poor solubility in aqueous environments or, if they are soluble, face rapid elimination from the bloodstream through filtration in the kidney. In the past, efforts have been made to modify the molecule itself, often following the "rule-of-five" guidelines developed by Lipinski to raise awareness of the properties and structural features that render molecules more or less "drug-like" [60]. Dendrimers present an attractive alternative strategy to the redesign of the drug because they allow unfavorable properties of a small molecule, such as insolubility, to be overcome by the larger characteristics of the macromolecule. An approach for improving the pharmacological properties of higher molecular weight drug candidates, analogous to Lipinski's guidelines for the modification of small molecule drugs, has been applied for protein therapeutics such as recombinant antibodies 
and protein toxins used in cancer treatment. In these cases, the amino acid sequences of recombinant proteins have been "humanized" by genetic engineering to avoid immunogenicity $[61,62]$ and their glycosylation patterns have been modified to increase serum half-life $[63,64]$. These efforts, undertaken with actual proteins, illuminate design features that can benefit the development of protein mimics, dendrimers. In particular, the "humanizing" experiments show that small changes, such as the substitution of a single amino acid for another, can avoid significant problems like undesired systemic immune responses. In the same manner, small changes in the surface properties of dendrimers, such as the addition of poly(ethylene glycol) (PEG), can avoid unwanted immunogenicity. Finally, even extremely large therapeutic candidates, notably plasmids or naked viral DNAs used for non-viral gene delivery that are well beyond the size of traditional drugs, are also benefiting from dendrimer-assisted delivery. The next section outlines specific approaches for the delivery of both small and large drug candidates by dendrimers.

1.5 .2

Dendritic Drug Delivery: Encapsulation of Guest Molecules

\subsubsection{Dendrimers have Internal Cavities that can Host Encapsulated Guest Molecules}

The flexible branches of a dendrimer, when constructed appropriately, can provide a tailored sanctuary containing voids that provide a refuge from the outside environment [2] wherein drug molecules can be physically trapped [65] (Figs. 1.1 and 1.3). Encapsulation of hydrophilic, hydrophobic, or even amphiphilic compounds as guest molecules within a dendrimer [66] can be enhanced by providing various degrees of multiple hydrogen bonding sites or ionic interactions $[65,67]$ or highly hydrophobic interior void spaces $[68,69]$. A wide variety of molecules have been successfully encapsulated inside dendrimers. In early experiments, compounds used to demonstrate the "guest molecule" concept included easy-to-visualize dye molecules such as rose bengal [66] and Reichardt's dye [69] as well as pyridine [65] and peptides [67]. More recently, actual drugs, including 5-fluorouracil [70], 5-amino salicylic acid, pyridine, mefanminic acid and diclofenac [65], paclitaxel [71, 72], docetaxel [73], as well as the anticancer agent 10-hydroxycamptothecin [69], have been successfully encapsulated. Together, these results demonstrate that encapsulation is a general strategy for the delivery of low molecular weight compounds by dendrimers. This method is anticipated to be of particular value when display of the bioactive molecule on the surface of the dendrimer induces unwanted immunogenicity or reduces biocompatibility (Section 1.5.5).

\subsubsection{Using Dendrimers for Gene Delivery}

The delivery of small molecules complexed as guest molecules in internal void spaces of dendrimers is, at least in retrospect, intuitively obvious. By contrast, the delivery of extremely large macromolecules, such as MDa-sized plasmid DNA for non-viral gene therapy, is counter-intuitive because the encapsulation of a "guest" molecule many times the molecular weight of the dendrimer itself appears impos- 
sible. Nonetheless, experimental evidence had demonstrated that gene delivery strategies also benefit from the participation of dendrimers [74]. For example, from its original discovery of efficacy for gene delivery [75], the fractured form of PAMAM, known as Superfect ${ }^{\mathrm{TM}}$, is now a commercially-available transfection agent for in vitro applications [76]. Typical approaches to optimize dendritic gene delivery for in vivo use have involved the surface modification of a PAMAM backbone, either with arginine [77] or hydroxyl groups [78]. Alternatively, the results reported by Kim and coworkers, who demonstrated improved gene delivery with a novel PAMAM-PEG-PAMAM triblock copolymer, show that construction of dendrimers composed of new building blocks is warranted [76]. Although still in their infancy, there are efforts afoot to exploit dendrimers for the delivery of smaller nucleic acids such as antisense oligonucleotides and short interfering RNAs (siRNA); the success of these applications is likely to depend on the continuing development of novel materials for dendrimer synthesis [79].

\subsubsection{Release of Encapsulated "Pro-drugs"}

Once a dendrimer carrying an encapsulated drug reaches the intended site of action, the guest molecule generally must be released to gain bioactivity. Indeed, a concern is that the active drug would "leak" out prematurely, thereby reducing the amount available for the intended therapeutic intervention, or more ominously, result in systemic toxicity. Reassuringly, early experiments showed that the close packing of dendritic branches on the surface of the macromolecule (Fig. 1.1) effectively formed a "membrane" that reduced diffusion to immeasurably slow rates [66]. In other cases, the release of encapsulated guest molecules was relatively faster, occurring over a few hours, apparently through hydrolytic degradation of the dendrimer in aqueous conditions [65]. The observation that guest molecules could be liberated at different rates demonstrated that viable opportunities exist to tailor the release for either slow or rapid delivery (Fig. 1.5). At present, additional control of delivery rates is being sought; for instance, the ability of a dendrimer to instantaneously release its entire drug payload upon reaching its cellular target would be valuable. Promising steps in this direction are being taken by the development of $\mathrm{pH}$-sensitive materials [65], the fine tuning of hydrolytic release conditions, and the selective liberation of guest molecules on the basis of their size or shape [80].

\subsection{3}

\section{Covalent Conjugation Strategies}

\subsubsection{Dendrimers Overcome many Limitations Inherent in Polymeric Conjugation Strategies}

The strategy of coupling small molecules to polymeric scaffolds by covalent linkages to improve their pharmacological properties has been under experimental test for over three decades [81-84]. Unfortunately, conventional linear polymers typically used in these efforts are plagued by inherent properties that render them distinctly "un-drug-like", including high polydispersity and size distributions, a 


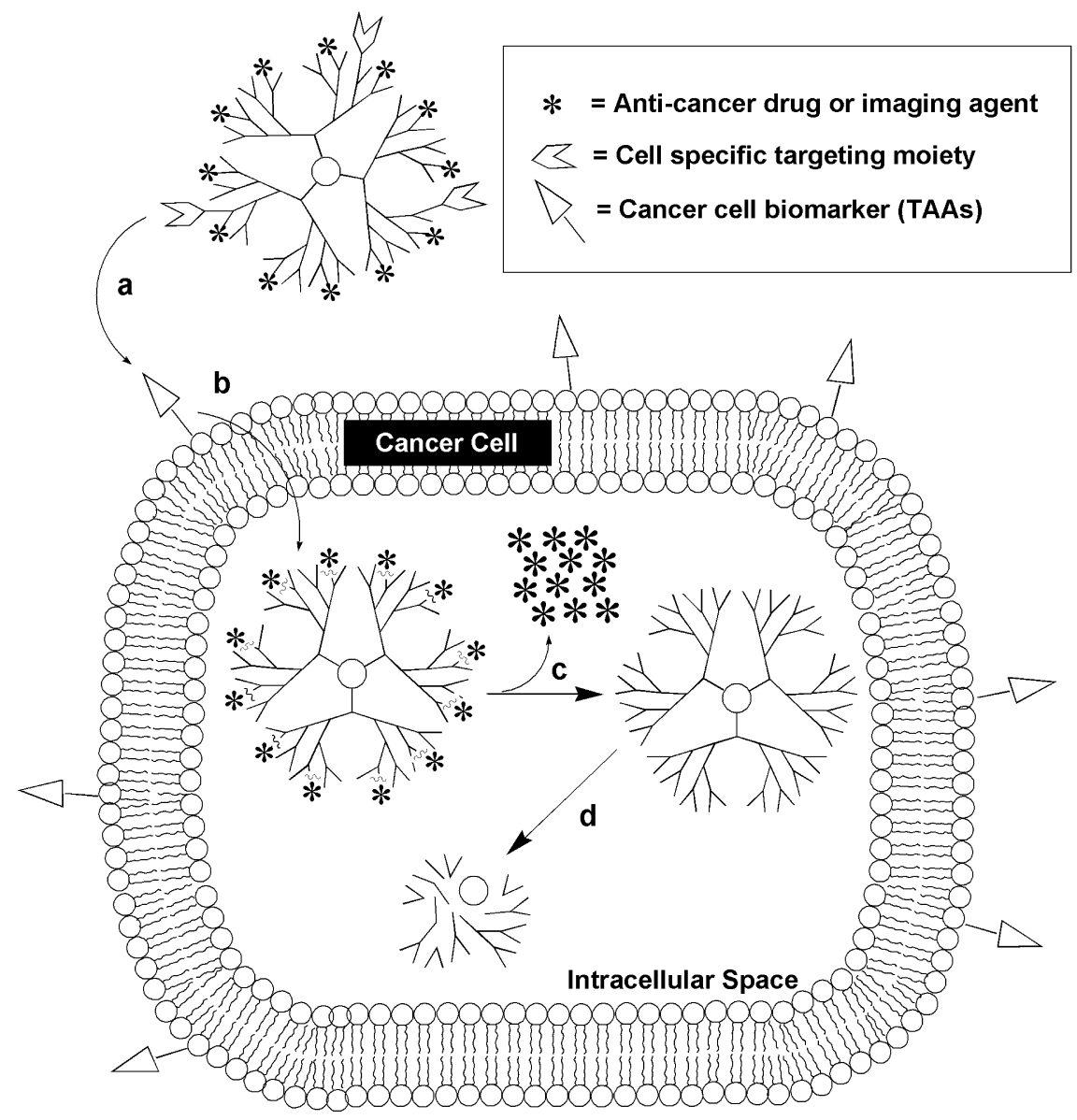

Fig. 1.5. Requirements for dendrimer-based, cancer-targeted drug delivery. (a) Dendrimers with multiple surface functional groups (Section 1.4.3) can be directed to cancer cells by tumor-targeting entities that include folate or antibodies specific for tumor-associated antigens (TAAs). (b) The next step is intake into the cell, which in the case of folate targeting occurs by membrane receptormediated endocytosis (Section 1.6.3.2). (c) Once inside the cell, the drug generally must be released from the dendrimer, which, for the self-immolative method (Section 1.5.3.3), results in the simultaneous disintegration of the dendritic scaffold (d).

lack of defined structure, and a low density of drug payload per unit volume or mass. Properties of dendrimers that overcome these problems include monodispersity that results in the ability to select the precise sizes of nanoparticle required to a specific application (Table 1.1), a fully defined structure that allows the presentation of attached conjugates in a defined architecture, a high ratio of drug payload to volume, and enhanced control over drug release rates. Unsurprisingly, based on these many beneficial features, a wide range of biologically active molecules have 
already been covalently attached to dendrimers. These conjugates range from small molecule drugs, such as ibuprofen [85], fluorescent and radioactive imaging agents (Section 1.6.4), oligonucleotides, oligosaccharides and peptides, as well as much larger molecules such as monoclonal antibodies (Section 1.6.3). Biologically active molecules attached to dendrimers can have two fundamentally different relationships to the host molecule. In some cases, exemplified by vaccine applications, there is no need to liberate active drug from the dendrimer (indeed, the success of antibody production usually depends on the unique display characteristics achieved by conjugation to the dendrimer). In most cases, however, the conjugated dendritic assembly functions as "pro-drug" where, upon internalization into the target cell, the conjugate must be liberated to activate the drug.

\subsubsection{Dendrimer Conjugates can be Used as Vaccines}

Most low molecular weight substances are not immunogenic; consequently, when it is desired to raise antibodies against small molecules, they must be conjugated to a macromolecule. In the past, natural proteins have commonly been used as carriers to generate antibodies to small molecules; now an alternative strategy using dendrimers has been demonstrated. In particular, unmodified PAMAM dendrimers that fail to elicit an antibody response on their own become haptenized upon protein conjugation and generate a dendrimer-dependent antigenic response $[86,87]$. A specific example of this technique is provided by the dendrimeric presentation of antigenic HIV peptides, which proved superior to other multimeric presentation strategies, such as conjugation to dextran [88]. Notably, the immunogenicity of dendrimer conjugates is not limited to peptides antigens; in one study antibodies were produced against densely penicilloylated dendrimers that were subsequently used for the diagnostic testing of patients with potential allergy to $\beta$-lactam antibiotics [89]. Finally, although carbohydrate-conjugated dendrimers (Section 1.4.3.2) are typically non-immunogenic [1], antibodies can be successfully elicited against cancer-specific oligosaccharides displayed on a dendritic scaffold, offering a method for generation of a new class of cancer vaccines (Section 1.6.6.2).

\subsubsection{Release of Covalently-delivered "Pro-drugs"}

Similar to encapsulated guest molecules that generally require release from the void spaces of a dendrimer to gain bioactivity (Section 1.5.2.3), a covalently delivered dendritic conjugate must also be cleaved within the target cell to regenerate the active cytotoxic agent (Fig. 1.5). At the same time, to ensure systemic nontoxicity, the covalent linker must be stable in circulation [90]. Several strategies are being pursued to ensure the successful cleavage and activation of the pro-drug in the target cell or tissue while avoiding systemic release. These include activation by low $\mathrm{pH}$ found in endosomal vesicles, installation of enzyme-cleavable ester linkages into the linkers that attach the pro-drug to the dendritic macromolecule, or disulfide bonds that are liberated in the reducing environment of the endoplasmic reticulum, photoactivation, or sensitivity to ultrasound [1].

Briefly returning to the benefits of dendritic clusters over conventional polymers for drug delivery, problems with the delivery of covalent conjugates when conven- 
tional polymers, such as poly(lactic acid) (PLA) or its copolymer with glycolide (PLGA), are used include a lack of sustained drug release [91]. Generally, these and other linear, randomly oriented polymers have an initial burst where as much as $50 \%$ of drug is released followed by a dramatic drop-off. An advantage of dendrimers is that their release rates are more consistent, which has been demonstrated by polylactide-PAMAM dendrimers [91] and dendrimer-platinate [92]. Consistent release from dendrimers is likely an inherent feature of their defined three-dimensional structure as their sites of drug attachment are continuously exposed to solvent, compared with random polymers where conjugated pro-drug moieties can be internalized randomly. The unique architectural features of dendrimers offer additional elegant strategies to gain exquisite control over release of active drug. In particular, the production of dendrimers functionalized with catalytic antibodies [68] has spurred the development of dendrimers capable of "selfimmolation" [93-95].

Self-immolative dendrimers provide an attractive potential platform for multidrug delivery. To briefly explain, these unique assemblies have the ability to release all of their tail units (i.e., the active drug) through a self-immolative chain fragmentation, which is initiated by a single cleavage at the dendrimer's core [96]. The first generation of dendritic prodrugs was demonstrated by Shamis and coworkers who synthesized doxorubicin and camptothecin as tail units and designed a retro-aldol retro-Michael focal trigger provided by action of the catalytic antibody 38C2 [94]. This method showed a dramatic increase in toxicity to tumor cells upon bioactivation of the pro-drug compared with tests done in the absence of the activating antibody. This technology, when fully developed into a complete chemical adaptor system that combines a tumor-targeting device (Section 1.6.3), a pro-drug, and pro-drug activation trigger, provides a sophisticated platform for future research efforts and the development of drugs for in vivo use [93].

\subsection{4}

\section{Fine-tuning Dendrimer Properties to Facilitate Delivery and Ensure Bioactivity}

\subsubsection{Delivery Requires Avoiding Non-specific Uptake}

From the initial entry into the body, a drug candidate confronts many barriers and diversions on its route to the site of intended bioactivity. Uptake by oral ingestion is ideal for patient comfort and, while still largely speculative for dendrimers [97], there is now evidence that uptake occurs in the rat gut [98]; this route is enticing based on an increasing recognition that nanoparticle uptake across the gut is largely governed by the physicochemical properties and surface chemistries of oral drug delivery vehicles [99]. Typically, to get to the target site in the body, the drug candidate must avoid becoming trapped with the extracellular matrix, which has been shown to hinder cellular uptake and reduce the efficiency of other nanosized delivery vehicles [100]; instead entry into the bloodstream is generally required for transit to the intended site of action.

Once in the bloodstream, either by successful navigation of an oral route or through direct injection, dendrimers below a certain size are at risk of filtration 
and removal by the kidney. This pitfall, however, can be avoided by ensuring that sufficiently large dendrimers are used. Indeed an important design feature and overriding impetus to use dendrimer delivery vehicles is to prevent the filtration of these drug candidates by the kidney. A second, off-target "trap" for dendrimers has been identified in a study that showed sequestration of dendrimers in the liver and spleen, in part due to their surface properties and in part due to their size [101]. As discussed elsewhere, both of these parameters can be controlled with exquisite sensitivity for dendritic macromolecules, allowing longer residence times in the blood (the longer the serum half-life, the greater the opportunity to reach the intended site of action).

\subsubsection{2 “Local" Considerations: Contact with, and Uptake by, the Target Cell}

Once a dendrimer has successfully entered the bloodstream and has been designed to minimize undue accumulation in non-target organs or tissues, it still faces the challenge of seeking out and interacting with its targeted site of action. The diversity of cell surface targets available for a nanodevice to bind to is vast; here we limit ourselves to specific examples related to cancer (Section 1.6.3). We will jump ahead to the point when a dendrimer has made "first contact" with a cell and reflect on how it interacts with the membrane. In this regard, there are provocative studies with PAMAM polymers that suggest that binding to the cell surface is facilitated by the deformable properties of dendrimers [15, 16, 18] (Section 1.3.1.3). Cellbinding induced deformations, if they prove real, have important implications for drug delivery. For example, the flattened forms of dendrimers lose their internal voids where guest molecules - such as drug payloads - are sequestered [6]. If this step occurs too soon, i.e., outside of the target cell, the drug might be ineffective, whereas if it occurs at the right moment, i.e., in the cytosol for cytosolic-acting drugs, it would provide an additional design parameter to exploit in the drug release process (Section 1.5.2.3). Notably, the deformations proposed to occur upon the interaction of a dendrimer with a cell, where the dendrimer shifts from a canonical "spherical" shape to a flattened disk with a significant loss in volume, have been most-extensively investigated at the dendrimer-mica interface. Clearly, the plasma membrane of a cell shares few biophysical characteristics with an extremely flat and rigid surface of mica, therefore, combined with the thermodynamically unfavorable aspects of the putative shape change, the extrapolation to drug delivery in biological systems should not be overstated. Encouragingly, shape changes also have been observed - but not thoroughly characterized - for dendrimers encountering the air-water interface, which is a better model for biological systems. Regardless of the current lack of concrete information, the intriguing nature of this potential mechanism for cell targeting and drug release merits its discussion here and also warrants further experimental investigation.

Once a dendrimer is in contact with a cell, there is strong experimental evidence that the exact surface properties of the dendrimer influence cellular uptake [102]. Therefore, the ability to modulate the chemical properties of a dendrimer provides additional options for controlling the uptake of a dendritic drug delivery device into a cell and even partitioning pro-drug release into specific organelles. To elaborate 
by briefly recapitulating a series of elegant experiment from the Banaszak Holl group, these researchers used a battery of assays, ranging from dye leakage to atomic force microscopy, to demonstrate that G5-G7 PAMAM dendrimers disrupt lipid bilayers and form holes large enough $(5-40 \mathrm{~nm})$ to account for dendrimer internalization. Moreover, the hole formation could be tuned by the exact size of the dendrimer, as well as surface chemical properties. To be specific, G7 amineterminated PAMAM initiated hole formation while its G5 counterpart did not. The smaller G5 dendrimer, however, did expand holes at existing defects; by contrast, acetamide terminated G5 PAMAM neither initiated hole formation nor expanded existing defects $[102,103]$. The mechanism of hole formation in membranes by PAMAM was proposed to involve the removal of lipid molecules from the membrane to form aggregates consisting of a dendrimer surrounded by lipid molecules [103]. Once inside a cell, there are early indications that the precise properties of a dendrimer can influence subcellular trafficking. Eventually, if these processes can be better understood and controlled, their exploitation for drug delivery will be very attractive considering that some entities, such as dendrimerdelivered ibuprofen, need to only gain access to the cytosol [85], whereas other class of drugs, such as dendrimer-delivered plasmid DNAs, have the moredemanding task of reaching the nucleus [104].

1.5 .5

Drug Delivery: Ensuring the Biocompatibility of Dendritic Delivery Vehicles

\subsubsection{Biocompatibility Entails Avoiding "Side Effects" such as Toxicity and Immunogenicity}

To briefly reiterate, properties of dendritic polymers important for drug delivery include negligible polydispersity, a high-density payload of pro-drug, and the ability to selectively release the active form of drug precisely at its intended site of action. Although dendrimers are capable of each of these tasks, their advantages are for naught if the final dendritic complex is not "biocompatible." Biocompatibility, a broad term with numerous meanings, will be considered here from three perspectives, water solubility, lack of immunogenicity, and toxicity.

\subsubsection{Water Solubility and Immunogenicity}

The first two biocompatibility issues mentioned above, namely water solubility and immunogenicity, are closely related insofar as highly-hydrated macromolecules tend to be less immunogenic. With dendrimers, there are many options available to overcome difficulties that arise in these areas. For example, solubility can be readily adjusted by surface modifications to surface chemistry or by the addition of conjugated ligands (Section 1.5.3, Fig. 1.3). Moreover, dendrimers such as the commonly used G3, G5, and G7 PAMAM clusters are not inherently immunogenic [105]. Derivatized PAMAM such as the G4D-(1B4M-Gd) 62 magnetic resonance imaging (MRI) contrast dendrimer, however, can become immunogenic (which is not surprising considering the deliberate efforts to render small molecules immunogenic through presentation on a dendritic scaffold). This problem - 
once again tying together the concepts of solubility and immunity - was overcome in one study by conjugation of poly(ethylene glycol) (PEG) to the surface of the dendrimer. Notably, PEG also had the positive effect of decreasing non-specific clearance from the blood, likely due to the increased hydration and resulting solubility of the particle [106].

\subsubsection{Inherent and Induced Toxicity}

A basic issue in drug delivery is the avoidance of non-specific, systemic, or offtarget toxicity. At its simplest this issue, when applied to dendrimers, involves the biological effects of the material used to construct the polymer. Ideally, the building blocks themselves, as well as their degradation products upon delivery and release of the drug payload, are non-toxic. One strategy is to directly use natural biological molecules, such as carbohydrates [59, 107], amino acids and peptides [108], nucleic acids [109-113], or lipids [114, 115] as the building blocks. To provide additional synthetic flexibility, while maintaining biocompatibility, an increasing number of biologically compatible and generally-regarded as safe (GRAS) materials are being used in dendrimer construction. Examples include dendritic polyglycerol [116], melamine [117]; phosphate [118], polyglycerols [39], a polyester dendrimer based on poly(ethylene oxide) that has tunable molecular weights and architectures [84], and dendrimers composed of citric acid and poly(ethylene glycol) [65].

The pioneering PAMAM-based dendrimers illustrate a second issue beyond inherent toxicity of the material or breakdown products, namely "induced" toxicity. The PAMAM family (Table 1.1), although not explicitly designed for biocompatibility, was found to be non-toxic when generations 1 through 5 were tested [105]. Evaluation of G7 dendrimers, however, showed potential biological complications, including dose-dependent toxicity [105], thereby illustrating that, while the basic material of PAMAM is inherently non-toxic, deleterious outcomes could be "induced" by factors such as the size or structure of the nanodevice. Smaller generation, non-toxic, dendrimers are sufficient for some applications but larger clusters are needed to fully exploit the enhanced permeation and retention (EPR) effect important in the treatment of cancer with macromolecular therapeutics (Section 1.6.2); consequently, toxicity cannot simply be avoided by restricting use to small, safe-sized particles. Instead, one strategy devised to avoid toxicity was the re-design of the building blocks of PAMAM-based material [76, 119] while another strategy involved the development of completely new polymeric backbones [120].

The selection of "safe" building blocks to avoid deleterious effects in dendrimer construction is unlikely to prevent all problems. To illustrate, even very safe building blocks, such as amino acids, can be highly toxic or immunogenic when assembled into large macromolecules - in this case proteins - in the "wrong" way. Indeed, the toxicity of dendrimers could be the result of several factors beyond the simple properties of the unloaded scaffold. For instance, with cancer drugs intended to kill cells, systemic toxicity could result if the drug is taken up by the wrong cellular target (i.e., a healthy cell or tissue, rather than a cancer cell or tumor) or if the nanodevice was "leaky" (i.e., if the pro-drug was released systemically before reaching the target cell). Fortunately, many strategies exist for prevent- 
ing toxicity, including directing a drug to its intended site of action by targeting moieties (Section 1.6.3) and developing sophisticated release strategies (Section 1.5.3.3). Problems that arise from the surface properties of the conjugated dendrimer can be ameliorated by masking the surface with something as simple as PEG or, in more advanced schemes, by coating with sugars or peptides to make glycodendrimers or peptide dendrimers, respectively (discussed in Ref. [121]) to mimic proteins naturally found in circulation (Section 1.4.3.2).

1.6

Dendrimers in Cancer Diagnosis and Treatment

1.6.1

Dendrimers have Attractive Properties for Cancer Treatment

Cancer epitomizes the challenges faced during drug delivery: an anticancer drug must be able to seek out subtle changes that distinguish a transformed cell from the other 200 or so types of healthy cells found in the body and then provide a sufficiently high dose of a toxic agent to selectively kill the cell while not harming its healthy neighbors. Therefore, even though dendrimers can be endowed with many favorable properties for drug delivery (Section 1.5), an ultimate challenge - ergo, a "real-world" test - of these versatile nano-devices will be whether they can successfully meet the formidable tasks of diagnosing and treating of malignant disease. As described in Section 1.7, although significant work remains in several areas, prospects now appear bright for dendrimer-based approaches to cancer treatment.

1.6.2

Dendrimer-sized Particles Passively Accumulate at the Sites of Tumors

To begin the discussion of properties that make dendrimers attractive vehicles for cancer treatment, we revisit the concept that encapsulation (Section 1.5.2) or covalent linkage (Section 1.5.3) of small molecule drug candidates to a dendrimer enhances the pharmacological properties of the drug. In cancer chemotherapy, these desirable size-based features are reinforced by the enhanced permeability and retention (EPR) effect that improves the delivery of macromolecules to tumors. The EPR effect is based on unique pathophysiological features of a solid tumor, such as extensive angiogenesis resulting in hyper-vascularization, limited lymphatic drainage, and increased permeability to lipids and macromolecules. These features, which help ensure adequate nutrient supply to meet the metabolic requirements of rapidly growing tumors $[122,123]$, can be turned to the tumor's disadvantage by the use of nano-sized therapeutic agents.

The EPR effect was discovered when selective accumulation of the SMANCS conjugate (styrene-maleic anhydride-neocarzinostatin) was observed at the site of tumors while similar accumulation was not seen with neocarzinostatin alone 
$[124,125]$. The EPR response was subsequently demonstrated for similarly-sized liposomes, thereby establishing that this effect was largely a function of particle size and did not solely depend on the chemical or biophysical properties of the macromolecule. Specifically, in one study optimal tumor delivery occurred for liposomes having a size distribution between 70 and $200 \mathrm{~nm}$ in diameter [126]. An independent study showed efficacy for liposomes loaded with daunorubicin in the same size range; specifically, those $\sim 142 \mathrm{~nm}$ in diameter exhibited an inhibitory effect against Yoshida sarcoma whereas smaller $(\sim 57-58 \mathrm{~nm})$ and larger $(\sim 272$ $\mathrm{nm}$ ) liposomes had weaker or no effect [127]. Over time, cautionary notes were raised that tempered initial enthusiasm for exploiting the EPR effect for cancer treatment. For example, the porosity of the vasculature in tumors can be highly variable even with a single vessel that can be leaky to one size of particle in one region but not in another [128]. Experimentally addressing this issue was complicated by the size polydispersity of traditional nanoparticles used to exploit the EPR effect, which were typically either lipids or conventional polymers that rendered a significant proportion of intended drug inactive. Fortunately this issue - the ability to match exact and uniform sizes needed to target an individual tumor - is highly tractable with dendrimers because selection of an exactly-sized entity is possible (Table 1.1) compared with the large size distributions that plague liposome and most polymeric materials [42].

The ability to construct monodisperse populations of dendrimers in the size range needed to exploit the EPR effect is an encouraging step towards the passive exploitation of tumor properties. Once the basic issue of size was resolved, however, secondary challenges (and opportunities) arose from observations that the chemical properties of the nano-sized particle can play significant roles in modulating the EPR effect. By way of a specific example, "conventional" polymeric materials showed efficacy at a smaller size range, occurring at $\sim 60 \mathrm{~nm}$ for both watersoluble and hydrogel forms of poly(vinyl alcohol) (PVA) [129], whereas almost identically-sized $57 \mathrm{~nm}$ egg phosphatidylcholine (EPC)-liposomes were ineffective [127]. As reported above, liposomes about twice this size showed maximal efficacy, so it was not unexpected that the EPC-liposomes were ineffective. Interestingly, however, hydrogenated egg phosphatidylcholine (HEPC)-liposomes in this size range (specifically, $58 \mathrm{~nm}$ ) were active [127], illustrating that the exact chemical properties of the material is a critical design parameter. In this respect, the many options for dendrimer "building blocks", as well as the ability to further tune surface properties provide many opportunities to endow dendrimers with favorable "passive" properties for tumor targeting.

1.6 .3

Multifunctional Dendrimers can Selectively Target Biomarkers found on Cancer Cells

\subsubsection{Methods for Targeting Specific Biomarkers of Cancer}

As discussed above, dendrimers can achieve passive EPR-mediated targeting to a tumor simply by control of their size and physicochemical properties. Passive tar- 
geting, which localizes the nano-particle in the close vicinity of a cancer cell, can be immediately useful for diagnostic purposes (Section 1.6.4) or for the delivery of radioisotopes capable of killing any cell within a defined radius. In general, however, most delivery strategies require that the anticancer agent directly attached to, or be taken up by, the target cell. The ability to append more than one type of functionality to a dendrimer (Fig. 1.3) allows the inclusion of ligands intended to bind specifically to cancer cells in the design of a multi-functional drug-delivery nanodevice (Fig. 1.5). Although a wide range of targeting ligands have been considered, including natural biopolymers such as oligopeptides, oligosaccharides, and polysaccharides such as hyaluronic acid, or polyunsaturated fatty acids $[90,130]$, discussion here is limited to folate, which is an exemplary small molecule tumor-targeting agent [42], as well as monoclonal antibodies directed against tumor associated antigens (TAAs).

\subsubsection{Targeting by Folate, a Small Molecule Ligand}

Folate is an attractive small molecule for use as a tumor targeting ligand because the membrane-bound folate receptor (FR) is overexpressed on a wide range of human cancers, including those originating in ovary, lung, breast, endometrium, kidney and brain [131]. As a small molecule, it is presumed to be non-immunogenic, it has good solubility, binds to its receptor with high affinity when conjugated to a wide array of conjugates, including protein toxins, radioactive imaging agents, MRI contrast agents, liposomes, gene transfer vectors, antisense oligonucleotides, ribozymes, antibodies [131, 132] and even activated T-cells [133]. Upon binding to the folate receptor, folate-conjugated drug conjugates are shuttled into the cell via an endocytic mechanism, resulting in major enhancements in cancer cell specificity and selectivity over their non-targeted formulation counterparts [131, 132]. Recently, folate has been enlisted in an innovative dendrimer-based targeting schemes $([42,134]$, Section 1.6.6.1).

\subsubsection{Targeting by Monoclonal Antibodies}

Of the many strategies devised to selectively direct drugs to cancer cells, perhaps the most elegant (and demanding!) is the use of monoclonal antibodies that recognize and selectively bind to tumor associated antigens (TAAs) [135-138]. TAA-targeting monoclonal antibodies have been exploited as delivery agents for conjugated "payloads" such as small molecule drugs and prodrugs, radioisotopes, and cytokines [139, 140]. The field of "immunotherapy" envisioned almost a hundred years ago, and given renewed impetus a quarter century ago by the development of monoclonal antibody technologies, has nonetheless progressed erratically over the past two decades as many pitfalls have been encountered [139]. Current prospects remain mixed but hopeful; optimistically, progress marked by commercial interest with companies providing their immunotherapeutic drug candidates with flashy trademarked names, such as "Armed Antibodies"TM" [141]. Similarly, the rosy opinion that this field is "on the verge of clinical fruition" has been published recently [142]. Perhaps, more realistically, one recent synopsis 
holds out "hope" for a major clinical impact for this strategy within the next 10 years [136].

Although a detailed discussion of the many pitfalls encountered in immunotherapy efforts is beyond the scope of this chapter, one key issue - readily addressed by dendrimers - is the requirement that an extremely potent cytotoxic drug be used in targeted antibody therapy. This point is illustrated by the fact that the greatest progress in this field has occurred for immunotoxins, which are antibody-toxin chimeric molecules that kill cancer cells via binding to a surface antigen, internalization and delivery of the toxin moiety to the cell cytosol. In the cytosol, protein toxins, such as those from diphtheria or pseudomonas, catalytically inhibit a critical cell function and cause cell death [143]. The high potency of immunotoxins for killing cancer cells is dramatically illustrated by ricin, where the catalytic activity of this ribosome-inactivating enzyme allows a single immunotoxin conjugate to kill a cell upon successful uptake and trafficking to the site of action [144, 145].

A drawback of immunotoxins is their significant immunogenicity, which limits repeated use [136]; from a broader perspective, their repeated use is made necessary by difficulties in providing a sufficiently high drug load to eradicate all cancer cells despite the high potency of conjugated toxin. An alternative approach of radioimmunotherapy, where high energy radionuclides are conjugated to TAA-targeting antibodies, also shows promise [146] but suffers from indiscriminate toxicity (the surrounding healthy tissues, as well as off-target tissues, become irradiated in addition to the target cancer cells). A third possible approach for immunotherapy, the conjugation of commonly-used small molecule drugs to TAAs, is hindered by the relatively low potency of most low molecular weight therapeutics. To illustrate this point, $\sim 10000$ TAAs occur on a typical cancer cell [101], making this number the upper limit for the number of targeting antibodies that can bind to the cell. The widely used anticancer drug cisplatin, to give one example, requires internalization of at least $50 \times$ this level of drug molecules for therapeutic efficacy.

A numerical analysis of the cisplatin example presented above indicates that each tumor-targeting antibody would have to be modified with a large number of small molecules to be effective as an anticancer drug (in this case, roughly 50 cisplatin molecules upon superficial analysis). Modification of an antibody with multiple radioisotopes, toxins, or even small molecules to increase the efficacy of cell killing, however, diminishes or eliminates the inherent specific antigen-binding affinity of an antibody. Therefore, to maximize drug loading while minimizing the deleterious effects on the biological integrity of the host antibody, an attractive approach is to use a linker molecule, such as a dendrimer, that can be highly conjugated (or internally loaded) with drug while modifying only a single site on the surface of the antibody [147]. Methodology to covalently attach antibodies to dendrimers that preserve the activity of the antigen-antibody binding site [148, 149], e.g., by chemical modification of their carbohydrates and subsequent linkage to PAMAM [150], has opened the door for the inclusion of dendrimers in immunotherapy $[151,152]$, thereby enhancing the future prospects of this chronically "almost-there" strategy. 
1.6.4

Dendrimers in Cancer Diagnosis and Imaging

1.6.4.1 Labeled Dendrimers are Important Research Tools for Biodistribution Studies The synthetic ability to attach both a tumor-targeting antibody and a potent payload of anticancer drugs to the same dendritic molecule provides a platform for multifunctional nano-scale drug delivery devices (Fig. 1.5). Before this technology can be applied in the clinic, however, its safety and efficacy must be demonstrated; towards this end, fluorescently-modified dendritic conjugates have been used extensively to characterize cell targeting, surface binding, uptake and internalization, and even sub-cellular localization $[85,151,152]$. The radiolabeled counterparts appropriate for animal studies have allowed detailed examination of the biodistribution of dendrimers. Several radio-isotopes have been conjugated to dendrimers, including ${ }^{3} \mathrm{H}[153],{ }^{14} \mathrm{C}[105],{ }^{88} \mathrm{Y}[154],{ }^{111} \mathrm{In}[154,155]$, and ${ }^{125} \mathrm{I}[98,149,156-158]$. These studies have established that the chemical and physical properties of dendrimers can be tuned to favor distribution to or away from specific organs and, ultimately, to achieve favorable biodistribution to tumors. The methods used in these experiments, however, typically requiring post-administration dissection of the host animal to allow the analysis of organ sequestration and tissue distribution of the radioisotope, are clearly not applicable to clinical practice. Instead, they have served as an important stepping stone along the path towards non- or minimally-invasive diagnostic procedures, which are proceeding mainly by the development of MRI contrast agents.

\subsubsection{Towards Clinical Use: MRI Imaging Agents}

Upon successful demonstration of the selective accumulation of dendrimers at the sites of tumors in animal models, a natural extension of this approach was to substitute gadolinium for the previously-tested isotopes or fluorophores. Gadolinium $\left({ }^{153} \mathrm{Gd}\right)$ is the best known and most extensively utilized magnetic resonance (MR) contrast agent $[159,160]$ and has previously been shown to be valuable for the improved diagnosis of cancer [161, 162]. Importantly, the in vivo efficacy of gadolinium is greatly enhanced when used as part of a macromolecular system [159]; in the past, attempts to create macromolecular gadolinium platforms have included the conjugation of chelators for this metal to both proteins [163] and conventional polymers [164]. These efforts have met with mixed (but generally limited) success. By contrast, Kobayashi and Brechbiel report that, by conjugating gadolinium to dendrimers, the unique properties of these polymers, such as exquisite size control, allowed selective targeting and imaging of the kidney, vascular, liver, or tumors [159]. Of note, tumor specific targeting and accumulation of gadolinium contrast agents is possible by use of either the folate receptor [165] or TAAs [159]. A drawback of the initial PAMAM-based MR contrast agents was their long residence time in the body; this problem, however, can be met by modifying both the surface properties [106] and basic chemical composition of the dendrimer. Specifically, diaminobutane (DAB) dendrimer-based chelators were more rapidly excreted from the 
body, illustrating that the development of clinically-acceptable dendrimer MR platforms is realistic [166].

1.6 .5

Steps Towards the Clinical Realization of Dendrimer-based Cancer Therapies

\subsubsection{The Stage is now set for Dendrimer-based Cancer Therapy}

The use of dendrimers for cancer treatment is still in its infancy with few, if any, applications successfully translated to the clinic. Consequently, their use as diagnostic agents constitutes both an important goal in and of itself, and also a valuable "baby step" towards the ultimate goal of curing cancer. As discussed, the process of actual killing cancer cells entails the complicated process of drug uptake followed by release of the drug into the cytoplasm or nucleus and is clearly a more demanding process than cell surface labeling, or even localization to the vicinity of the tumor, sufficient for diagnostic purposes. Nonetheless, in some cases, the transition from imaging to therapy will be closely linked, as evidenced by efforts now underway to combine antibody-targeted MR imaging nanoparticles with the delivery of antiangiogenic genes intended to inhibit the vascularization to the V2 carcinoma model in rabbits [167]. Another promising strategy - boron neutron capture therapy - has undergone impressive development over the past decade and is presented next as a successful demonstration of the promise of dendrimer-based cancer therapies.

\subsubsection{Boron Neutron Capture Therapy}

Cisplatin-based therapies illustrate the need for multiple conjugations of small molecules - estimated at 50 for this platinum drug - to a targeting antibody (Section 1.6.3.3). While some efforts are underway to use dendrimeric strategies for platinum drug delivery [168], an even more demanding situation, where thousands of ligands are required per targeting antibody, is provided by boron neutron capture therapy (BNCT). Accordingly, BNCT will be discussed here as an illustrative example of how dendrimers can help overcome high hurdles in the development of innovative cancer therapies. As a brief background, BNCT is based on the nuclear reaction that occurs when boron-10, a stable isotope, is irradiated with low energy $(\leq 0.025 \mathrm{eV})$ or thermal neutrons to yield alpha particles and recoiling lithium-7 nuclei. A major requirement for the success of BNCT is the selective delivery of a sufficient number of boron atoms $\left(\sim 10^{9}\right)$ to individual cancer cells to sustain a lethal ${ }^{10} \mathrm{~B}(\mathrm{n}$, alpha $) \rightarrow{ }^{7} \mathrm{Li}$ capture reaction $[169,170]$. Considering that the maximal number of antigenic sites per tumor cell is in the range of 100000 , and more commonly only $1 / 10^{\text {th }}$ that level, an a priori calculation suggests that each targeting antibody must be linked to at least 2000, but preferably closer to 5000, boron atoms [101]. Clearly, a single TAA-targeting antibody cannot be directly conjugated at this level and conventional polymers - e.g., polylysine conjugated with $\sim 1700$ boron derivatives and linked to a targeting antibody - caused the antibody to lose in vivo tumor localizing properties [171]. By contrast, when a 
PAMAM dendrimer was used for polyvalent boron conjugation, the linked antibody maintained immuno-recognition (although in vivo tumor targeting remained problematic because the conjugated dendrimer had a strong propensity to mislocalize in the spleen and liver) [101]. Over the decade since these pioneering efforts were first reported, continued progress has been made to solve problems such as off-target tissue localization, which was traced to the size of the dendrimer and presence of a large number of amine groups on the surface of PAMAM, by exploiting the versatility of dendrimer chemistry. In short, the re-design of boronated, anti-body-targeted dendrimers has culminated in the successful treatment of gliomas in the rat $[158,169,172]$ and laid the foundation for translation of this technology into clinical tests in the foreseeable future.

1.6.6

Innovations Promise to Speed Progress

\subsubsection{1 “Mix-and-Match" Strategy of Bifunctional Dendritic Clusters}

Two lessons are immediately apparent from the dedicated efforts to bring dendrimer-based BNCT to fruition. One is that dendritic technologies, while still at an early developmental stage, hold tremendous promise and merit continued investigation. The second is that the coupling of one treatment modality (BNCT) with one targeting strategy (antibodies to a specific type of glioma) required a staggering amount of effort. The growing realization that cancer is hundreds, if not thousands, of unique diseases at the cellular and molecular level, suggests that a commensurate number of therapeutic strategies are needed. The diversity of targeting strategies (which are not limited to folate and TAAs discussed here), coupled with the many "payload" possibilities (beside radioisotopes, boron, and cisplatin discussed here) used to diagnose and kill cancer cells, means that there are literally tens of thousands of individually customized therapies required to fully confront the myriad clinical manifestations of cancer. The sobering reality is that, if each of these customized treatments will require a decade long effort by a large team of researchers and clinicians, the large problem of cancer treatment will not be solved for a long time.

Choi and coworkers [134] have come up with an innovative mix-and-match scheme that promises to offset this gloomy prediction. These researchers have recently reported a cancer-targeting strategy that is reminiscent of the antibodytoxin/immunoconjugate strategy where distinct, but linked, entities are used to first recognize and bind and then subsequently modify a cancer cell. Their strategy, however, has great potential to improve on both the "targeting" and "payload" aspects of cancer therapy by, at first seemingly paradoxically, completely dividing these functions into separate dendritic clusters (Fig. 1.6). The key to this approach was to include a DNA “zipper" on each dendrimer that allows the targeting cluster, composed of folate-derivatized PAMAM in proof-of-concept experiments [173], to be readily combined with the imaging or drug-carrying dendrimer by way of the complementary DNA strand [134]. It can be envisioned that the production of libraries of dendrimers targeted to different cancer-specific biomarkers can be pro- 


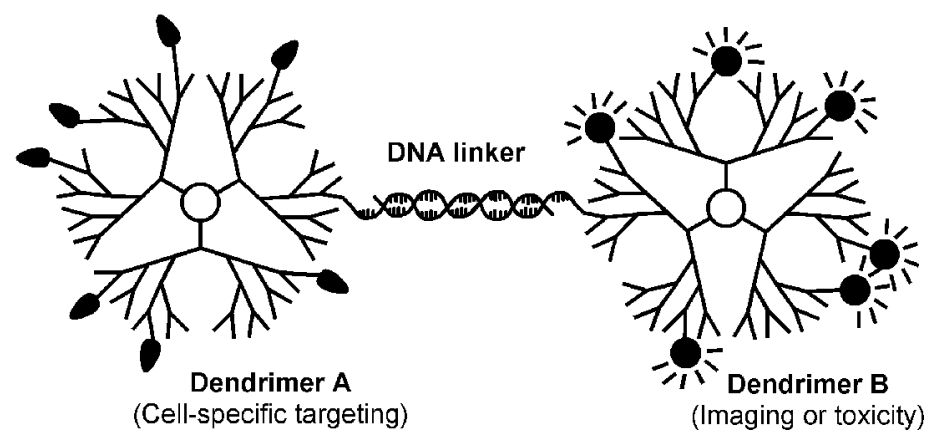

Fig. 1.6. DNA-dendrimer conjugates as potential cancer targeting imaging agents or therapeutics. (Adapted from Ref. [189].)

Differentially functionalized dendrimers covalently conjugated to complementary deoxyoligonucleotides can readily form duplex cancer cell-specific ligands hybridized to an imaging agent or drug. Cell-specific targeting ligands (e.g., folic acid in one study) are appended to Dendrimer A, and Dendrimer B is combinatorial nanoclusters that possess [134].

duced by a "mix-and-matched" strategy by combining "off-the-shelf" targeting and drug clusters as needed [42]. Development of easily-customizable nanomedicine platforms that exploit the facile duplex DNA formation for the generation of hybrid nano-clusters, thus circumventing the tedious synthesis of multiply-functionalized dendrimers, offers hope that the next ten years will witness rapid expansion of dendrimer technologies that build on the painstaking advances of the past decade.

\subsubsection{Towards Therapeutic Exploitation of Glycosylation Abnormalities found in Cancer}

Aberrant glycosylation, where the patterns of complex carbohydrate glycoforms found on the surfaces of cancer cells are dramatically different from those on healthy cells, is a hallmark of cancer [174-178]. Efforts to exploit these changes therapeutically, however, have long been stymied by the difficulty of controlling these complex and diverse molecules in an artificial synthetic setting. Today, with new technologies such as dendrimers that provide a platform for physiologicallyrelevant display of carbohydrates, new vistas are opening up for exploiting these molecules to intervene in malignant disease. Promising - but still early-stage efforts in this direction include the presentation of oligosaccharides found only in cancer cells $[53,56,58,179-181]$ on a dendritic scaffold (Section 1.4.3.2) for vaccine development (Section 1.5.3.2).

\subsubsection{Towards Targeting Metabolically-engineered Carbohydrate Epitopes}

As discussed above, one area of rapidly-expanding investigation is the abnormal glycosylation associated with the cancer cells; in particular dendrimeric scaffolds provide a unique platform to control the multimeric carbohydrate presentation needed to enact the "cluster glycoside effect" $[45,50,51]$, which is crucial for tar- 


\section{1 1 Dendrimers in Cancer Treatment and Diagnosis}

geting diseased tissues found in malignant diseases [1, 24]. Another approach to exploiting glycosylation for the treatment of cancer is through "chemical biology" strategies, such as the ability to express non-natural sialic acids on the cell surface through the use of $N$-acetylmannosamine (ManNAc) analogs [49, 182, 183] (Fig. 1.7). By appropriate design of the ManNAc analog, sialic acids, which are interesting nine-carbon sugars often overexpressed on cancer cells [175], can be provided with a "chemical handle" - such as a ketone, azide, or thiol [184-186] - for tar-
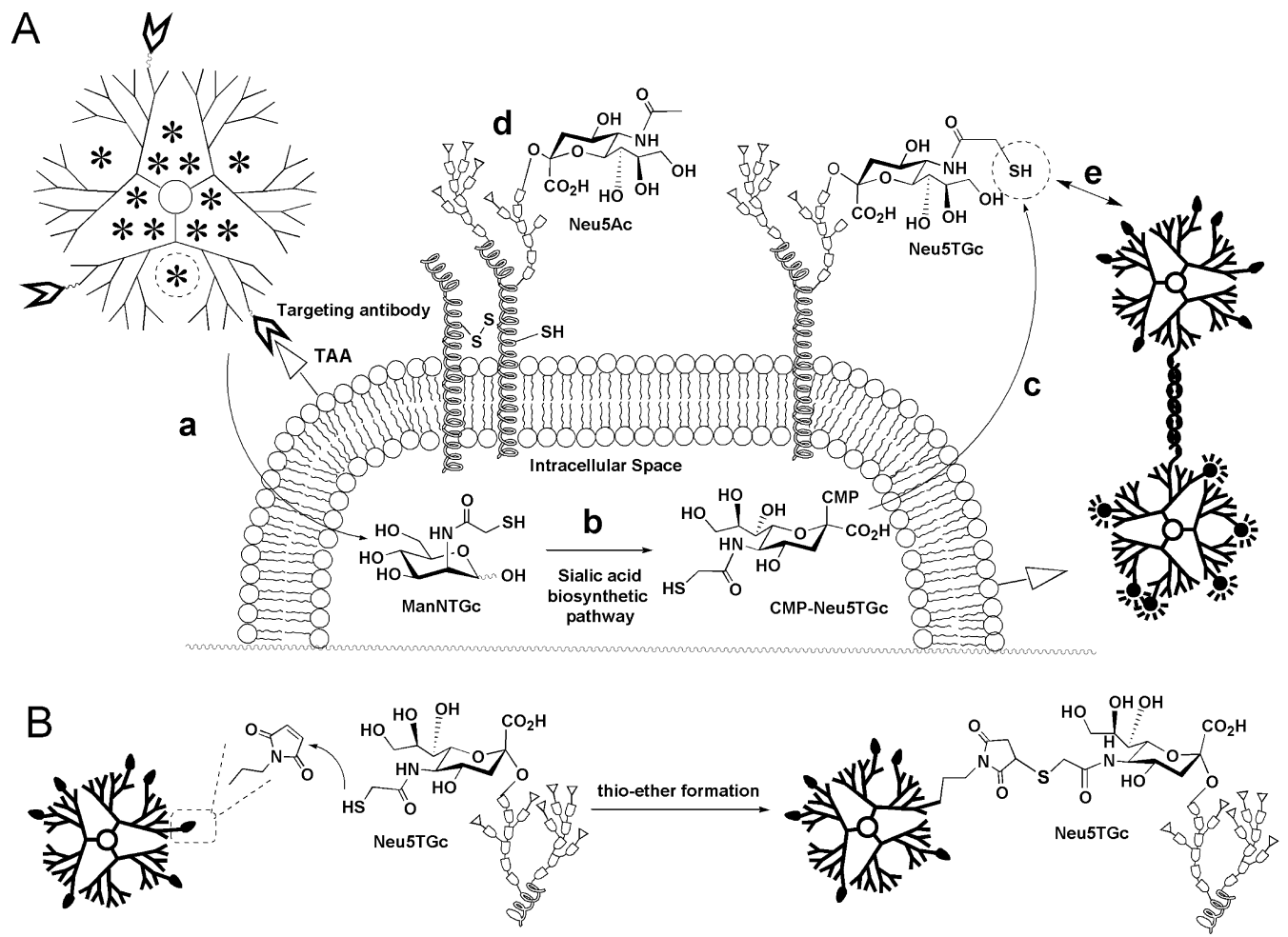

Fig. 1.7. Chemoselective targeting of drugloaded dendrimers to the cell surface. (A) Overview of sialic acid engineering. (a) A dendrimer can encapsulate and assist the delivery of $\mathrm{N}$-acetylmannosamine (ManNAc) analogs, such as the thiol-containing sugar "ManNTGc" (shown as " "**') into a cell (Section 1.5.2). (b) Once inside the cell, ManNTGc can be metabolically converted into CMP-Neu5TGc, a compound that serves as a sugar-nucleotide needed for the glycosylation process (c) where "Neu5TGc" a non-natural form of sialic acid, is installed into cell surface glycoconjugates. Overall, this process replaces natural sialic acids, such as "Neu5Ac", with their thiol-containing counterparts (d), which can then be targeted by dendritic assemblies such as the bifunctional "targeting" and "payload" clusters shown in Fig. 1.6. (B) Details of the "chemoselective ligation reaction" required for targeting the appropriately derivatized dendrimeric assembly to the cell. In this case, a maleimideconjugated targeting capsule will selectively interact with the sialic acid-display thiols to covalently bind the dendrimer to the cell surface via thio-ether bond formation. 
geted delivery of a second agent such as the ricin A-chain used in immunotoxins [187] or small molecule anticancer drugs [188]. Dendrimers offer assistance at several steps in this process of translating early-stage anticancer strategies like "sialic acid engineering" from the laboratory to clinical relevance. An enticing proposition is that the starting material - ManNAc, which like all sugars has notoriously poor pharmacological properties - can be made "drug-like" by encapsulation (or covalent ligation). Subsequently, after display of the target epitope on the cell surface, which is a modified thiol-bearing sialic acid in the case shown in Fig. 1.7, this can benefit from the high local density of dendritic display of maleimide to increase the rate of drug binding to the cell surface, which occurs over an unacceptably long period of several hours for current covalent coupling schemes [188]. This strategy, under evaluation in our laboratory, coupled with a high drug payload on the DNA-hybridized cluster (Fig. 1.6), provides renewed impetus for the already promising application of sugar-based therapeutic approaches to cancer. A particularly attractive aspect of this approach is that $\sim 10^{8}$ sialic acids exist on cancer cells, greatly improving prospects to deliver adequate levels of drug to achieve therapeutic efficacy compared with TAA-targeting schemes (Section 1.6.3).

\section{7}

\section{Concluding Remarks}

Dendrimers, chemically-defined entities with tunable biological properties, have advanced over the past two decades to the point where they stand on the cusp of major contributions to the treatment of cancer in a meaningful way. Although, as has been apparent by the many instances cited throughout this chapter where gaps in knowledge still remain and that must be plugged before dendrimers are ready for wide clinical use, their extreme versatility combined with the extensive research efforts now underway are sure to add sophistication to drugs already in use as well as spur the development of entirely new classes of anticancer therapy.

\section{Acknowledgments}

Funding was provided by the Whitaker Biomedical Engineering Institute and Department of Biomedical Engineering at The Johns Hopkins University, the Arnold and Mabel Beckman Foundation, and The National Institutes of Health.

\section{References}

1 U. Boas, P. M. H. HeegaArd, Dendrimers in drug research. Chem. Soc. Rev. 2004. 33, 43-63.

2 G. M. Dykes, Dendrimers: A review of their appeal and applications. $J$.
Chem. Technol. Biotechnol. 2001. 76, 903-918.

3 A. Sunder, J. Heinemann, H. Frey, Controlling the growth of polymer trees: Concepts and perspectives for 
hyperbranched polymers. Chem. Eur. J. 2000. 6, 2499-2506.

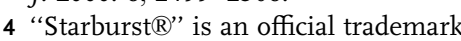
owned by Dow Chemical that covers PAMAM based dendrimers but the term "starburst" is often also used generically to describe the properties of certain dendritic macromolecules.

5 P. K. Maiti, T. ÇaGin, G. Wang, W. A. Goddard III, Structure of PAMAM dendrimers: Generations 1 through 11. Macromolecules 2004. 37, 6236-6254.

6 A. D. Schlüter, J. P. RABe, Dendronized polymers: Synthesis, characterization, assembly at interfaces, and manipulation. Angew. Chem. Int. Ed. 2000. 39, 864-883.

7 P. G. de Gennes, H. Hervet, Statistics of "starburst" polymers. J. Phys. Lett. 1983. 44, L351-L360.

8 B. KLAjnert, M. BRyszewska, Dendrimers: Properties and applications. Acta Biochim. Pol. 2001. 48, 199-208.

9 J. M. J. FrÉCHET, Functional polymers and dendrimers: Reactivity, molecular architecture, and interfacial energy. Science 1994. 263, 1710-1715.

10 C. L. Jackson, H. D. Chanzy, F. P. Booy, B. J. Drake, D. A. Tomalia, B. J. Bauer, E. J. Amis, Visualization of dendrimer molecules by transmission electron microscopy (TEM): Staining methods and Cryo-TEM of vitrified solutions. Macromolecules 1998. 31, 6259-6265.

11 D. K. Smith, F. Diederich, Functional dendrimers: Unique biological mimics. Chem. Eur. J. 1998. 4, 1353-1361.

12 S. Hecht, J. M. J. Fréchet, Dendritic encapsulation of function: Applying Nature's site isolation principle from biomimetics to materials science. Angew. Chem. Int. Ed. 2001. 40, 74-91.

13 A. Esposito, E. Delort, D. Lagnoux, F. Djojo, J.-L. Reymond, Catalytic peptide dendrimers. Angew. Chem. Int. Ed. 2003. 42, 1381-1383.

14 A. Clouet, T. Darbre, J.-L. Reymond, A combinatorial approach to catalytic peptide dendrimers. Angew. Chem. Int. Ed. 2004. 43, 4612-4615.
15 B. Chen, S. Piletsky, A. P. Turner, High molecular recognition: Design of "Keys". Comb. Chem. High Throughput Screen. 2002. 5, 409-427.

16 C. S. Goh, D. Milburn, M. Gerstein, Conformational changes associated with protein-protein interactions. Curr. Opin. Struct. Biol. 2004. 14, 104-109.

17 P. O. Glantz, T. Arnebrant, T. Nylander, R. E. Baier, Bioadhesion A phenomenon with multiple dimensions. Acta Odontol. Scand. 1999. 57(5), 238-241.

18 A. Mecke, I. Lee, J. R. Baker JR, M. M. Holl, B. G. ORR, Deformability of poly(amidoamine) dendrimers. Eur. Phy. J. E Soft Matter 2004. 14, 7-16.

19 D. Farin, D. Avnir, Surface fractality of dendrimers. Angew. Chem. Int. Ed. Engl. 1991. 30, 1377-1379.

20 S. L. Gilat, A. Adronov, J. M. J. FRÉCHET, Light harvesting and energy transfer in novel convergently constructed dendrimers. Angew. Chem. Int. Ed. 1999. 38, 1422-1427.

21 K. Autumn, Y. A. Liang, S. T. Hsieh, W. Zesch, W. P. Chan, T. W. Kenny, R. Fearing, R. J. Full, Adhesive force of a single gecko foot-hair. Nature 2000. 405, 681-185.

22 G. M. Dykes, L. J. Brierley, D. K. Smith, T. McGrail, G. J. Seeley, Supramolecular solubilisation of hydrophilic dyes by using individual dendritic branches. Chem. Eur. J. 2001. 7, 4730-4739.

23 R. Esfand, D. A. Tomalia, Poly(amidoamine) (PAMAM) dendrimers: from biomimicry to drug delivery and biomedical applications. Drug Discovery Today 2001. 6, 427436.

24 M. OzKan, Quantum dots and other nanoparticles: What can they offer to drug discovery? Drug Discovery Today 2004. 9, 1065-1071.

25 D. A. Tomalia, A. M. Naylor, W. A. GoddARD III, Starburst dendrimers: Molecular-level control of size, shape, surface chemistry, topology, and flexibility from atoms to macroscopic matter. Angew. Chem. Int. Ed. Engl. 1990. 29, 138-175. 
26 H.-B. Mekelburger, W. Jawarek, F. VöGTLE, Dendrimers, arborols, and cascade molecules: Breakthrough into generations of new materials. Angew. Chem. Int. Ed. Engl. 1992. 31, 1571-1576.

27 J. Issberner, R. Moors, F. Vögtle, Dendrimers: From generations and functional groups to functions. Angew. Chem. Int. Ed. Engl. 1994. 33 , 2413-2420.

28 D. GudAT, Inorganic cauliflower: Functional main group element dendrimers constructed from phosphorus- and silicon-based building blocks. Angew. Chem. Int. Ed. Engl. 1997. 36, 1951-1955.

29 M. Fischer, F. Vögtle, Dendrimers: From design to application - A progress report. Angew. Chem. Int. Ed. 1999. 38, 884-905.

30 G. R. Newrome, C. N. Moorefield, F. VöGTLE, Dendrimers and Dendrons: Concepts, Syntheses, Applications. 2001, Weinheim, Wiley-VCH.

31 S. M. Grayson, J. M. J. Fréchet, Convergent dendrons and dendrimers: From synthesis to applications. Chem Rev. 2001. 101, 3819-3868.

32 I. Gitsov, C. Lin, Dendrimers Nanoparticles with precisely engineered surfaces. Curr. Org. Chem. 2005. 9, 1025-1051.

33 N. Feuerbacher, F. Vögtle, Iterative synthesis in organic chemistry. Top. Curr. Chem. 1998. 197, 1-18.

34 E. Buhleier, W. Wehner, F. Vögtle, "Cascade"- and "nonskid-chain-like" synthesis of molecular cavity topologies. Synthesis 1978. 155-158.

35 G. R. Newkome, Z. Yao, G. R. Baker, V. K. Gupta, Micelles. Part 1. Cascade molecules: A new approach to micelles. A [27]-arborol. J. Org. Chem. 1985. 50, 2003-2004.

36 D. A. Tomalia, H. Baker, J. Dewald, M. Hall, G. Kallos, S. Martin, J. Roeck, J. Ryder, P. Smith, Dendritic macromolecules: Synthesis of starburst dendrimers. Macromolecules 1986. 19, 2466-2468.

37 D. A. Tomalia, H. D. Durst, Genealogically directed synthesis Starburst cascade dendrimers and hyperbranched structures. Top. Curr. Chem. 1993. 165, 193-313.

38 T. M. Miller, T. X. Neenan, Convergent synthesis of monodisperse dendrimers based upon 1,3,5trisubstituted benzenes. Chem. Mater. 1990. 2, 346-349.

39 R. HaAg, J.-F. Stumbé, A. Sunder, H. Frey, A. Hebel, An approach to core-shell-type architectures in hyperbranched polyglycerols by selective chemical differentiation. Macromolecules 2000. 33, 8158-8166.

40 C. J. Hawker, J. M. J. FréChet, Preparation of polymers with controlled molecular architecture. A new convergent approach to dendritic macromolecules. J. Am. Chem. Soc. 1990. 112, 7638-7647.

41 J. R. Morgan, M. J. Cloninger, Heterogeneously functionalized dendrimers. Curr. Opin. Drug Discov. Devel. 2002. 5, 966-973.

42 Y. Choi, J. R. BAKer JR, Targeting cancer cells with DNA-assembled dendrimers: A mix-and-match strategy for cancer. Cell Cycle 2005. 4, 669-671.

43 M. Meldal, P. M. St Hilaire, Synthetic methods of glycopeptide assembly, and biological analysis of glycopeptide products. Curr. Opin. Chem. Biol. 1997. 1, 552-563.

44 D. Zanini, R. Roy, Synthesis of new a-thiosialodendrimers and their binding properties to the sialic acid specific lectin from Limax flavus. J. Am. Chem. Soc. 1997. 119, 2088-2095.

45 K. J. Yarema, C. R. Bertozzi, Chemical approaches to glycobiology and emerging carbohydrate-based therapeutic agents. Curr. Opin. Chem. Biol. 1998. 2, 49-61.

46 J. J. Landers, Z. Cao, I. Lee, L. T. Piehler, P. P. Myc, A. Myc, T. Hamouda, A. T. Galecki, J. R. J. BAKER JR, Prevention of influenza pneumonitis by sialic acid-conjugated dendritic polymers. J. Infect. Dis. 2002. 186, 1222-1230

47 J. D. Reuter, A. Myc, M. M. Hayes, Z. GAN, R. Roy, D. Qin, R. Yin, L. T. Piehler, R. Esfand, D. A. Tomalia, J. R. BAKER JR, Inhibition of viral 
adhesion and infection by sialic-acidconjugated dendritic polymers. Bioconjugate Chem. 1999. 10, 271-278.

48 D. H. Charych, J. O. Nagy, W. SpeVAK, M. D. Bednarski, Direct colorimetric detection of a receptorligand interaction by a polymerized bilayer assembly. Science 1993. 261, 585-588.

49 O. T. Keppler, R. HorstKorte, M. Pawlita, C. Schmidt, W. Reutter, Biochemical engineering of the $N$-acyl side chain of sialic acid: Biological implications. Glycobiology 2001. 11, $11 \mathrm{R}-18 \mathrm{R}$

50 J. J. Lundquist, E. J. Toone, The cluster glycoside effect. Chem. Rev. 2002. 102, 555-578.

51 L. L. Kiessling, S. Pohl, Strength in numbers: Non-natural polyvalent carbohydrate derivatives. Chem. Biol. 1996. 3, 71-77.

52 W. B. Turnbull, J. F. Stoddart, Design and synthesis of glycodendrimers. J. Biotechnol. 2002. 90, 231255.

53 R. Roy, M. G. BAEK, Glycodendrimers: Novel glycotope isosteres unmasking sugar coding. Case study with Tantigen markers from breast cancer MUC1 glycoprotein. J. Biotechnol. 2002. 90, 291-309.

54 R. Roy, Synthesis and some applications of chemically defined multivalent glycoconjugates. Curr. Opin. Struct. Biol. 1996. 6, 692-702.

55 P. R. Ashton, S. E. Boyd, C. L BRown, N. JAYARAMAN, J. F. STODDART, A convergent synthesis of a carbohydrate-containing dendrimer. Angew. Chem. Int. Ed. Engl. 1997. 36, 732-735.

56 T. ToYokuni, S.-i. Накомori, A. K. Singhal, Synthetic carbohydrate vaccines: Synthesis and immunogenicity of Tn antigen conjugates. Bioorg. Med. Chem. 1994. 2, 11191132.

57 S. André, P. J. Ortega, M. A. Perez, R. Roy, H.-J. Gabius, Lactosecontaining starburst dendrimers: Influence of dendrimer generation and binding-site orientation of receptors (plant/animal lectins and immunoglobulins) on binding properties. Glycobiology 1999. 9, 1253-1261.

58 R. Roy, M.-G. Baek, K. RittenhouseOLson, Synthesis of N, $\mathrm{N}^{\prime}$ -

bis(acrylamido)acetic acid-based Tantigen glycodendrimers and their mouse monoclonal IgG antibody binding properties. J. Am. Chem. Soc. 2001. 123, 1809-1816.

59 M. M. Palcic, H. Li, D. Zanini, R. S. Bhella, R. Roy, Chemoenzymatic synthesis of dendritic sialyl Lewis(x). Carbohydr. Res. 1997. 305, 433-442.

60 M. S. Lajiness, M. Vieth, J. ERICKSON, Molecular properties that influence oral drug-like behavior. Curr. Opin. Drug Discov. Devel. 2004. 7 , 470-474.

61 N. R. Gonzales, R. De Pascalis, J. Schlom, S. V. S. Kashmiri, Minimizing the immunogenicity of antibodies for clinical application. Tumour Biol. 2005. 26, 31-43.

62 B. Matthey, A. Engert, S. Barth, Recombinant immunotoxins for the treatment of Hodgkin's disease (Review). Int. J. Mol. Med. 2000. 6, 509-514.

63 K. Viswanathan, S. Lawrence, S. Hinderlich, K. J. Yarema, Y. C. Lee, M. Betenbaugh, Engineering sialic acid synthetic ability into insect cells: Identifying metabolic bottlenecks and devising strategies to overcome them. Biochemistry 2003. 42, 15 215-15 225.

64 M. J. Betenbaugh, N. Tomiya, S. Narang, J. T. A. Hsu, Y. C. Lee, Biosynthesis of human-type N-glycans in heterologous systems. Curr. Opin. Struct. Biol. 2004. 14, 601-606.

65 H. Namazi, M. Adeli, Dendrimers of citric acid and poly (ethylene glycol) as the new drug-delivery agents. Biomaterials 2005. 26, 1175-1183.

66 J. F. G. A. JANSEN, E. M. M. DE BRABANDER-VAN DEN Berg, E. W. MeIjer, Encapsulation of guest molecules into a dendritic box. Science 1994. 266, 1226-1229.

67 U. Boas, S. H. M. Söntjens, K. J. Jensen, J. B. Christensen, E. W. Meijer, New dendrimer-peptide hostguest complexes: Towards dendrimers 
as peptide carriers. ChemBioChem 2002. 3, 433-439.

68 A. Córdova, K. D. Janda, Synthesis and catalytic antibody functionalization of dendrimers. J. Am. Chem. Soc. 2001. 123, 8248-8259.

69 M. T. Morgan, M. A. Carnahan, C. E. Immoos, A. A. Ribeiro, S. Finkelstein, S. J. Lee, M. W. GrinstafF, Dendritic molecular capsules for hydrophobic compounds. J. Am. Chem. Soc. 2003. 125, 15 48515489.

70 P. K. Tripathi, A. J. Khopade, S. Nagaich, S. Shrivastava, S. Jain, N. K. JAIN, Dendrimer grafts for delivery of 5-fluorouracil. Pharmazie 2002. 57, 261-264.

71 T. Ooya, J. Lee, K. Park, Effects of ethylene glycol-based graft, starshaped, and dendritic polymers on solubilization and controlled release of paclitaxel. J. Controlled Release 2003. 93, 121-127.

72 T. Ooya, J. Lee, K. Park, Hydrotropic dendrimers of generations 4 and 5 : Synthesis, characterization, and hydrotropic solubilization of paclitaxel. Bioconjugate Chem. 2004. 15, 1221-1229.

73 J. M. Benito, M. Gomez-Garcia, C. Ortiz Mellet, I. Baussanne, J. Defaye, J. M. Garcia Fernandez, Optimizing saccharide-directed molecular delivery to biological receptors: Design, synthesis, and biological evaluation of glycodendrimer-cyclodextrin conjugates. J. Am. Chem. Soc. 2004. 126, 10 355-10 363.

74 T. Hudde, S. A. Rayner, R. M. Comer, M. Weber, J. D. IsaAcs, H. Waldmann, D. F. LaRkin, A. J. George, Activated polyamidoamine dendrimers, a non-viral vector for gene transfer to the corneal endothelium. Gene Ther. 1999. 6, 939-943.

75 J. Haensler, F. C. Szoka Jr, Polyamidoamine cascade polymers mediate efficient transfection of cells in culture. Bioconjugate Chem. 1993. 4, 372-379.

76 T.-i. Kim, H. J. Seo, J. S. Choi, H.-S. Jang, J.-u. Baek, K. Kim, J.-S. Park,
PAMAM-PEG-PAMAM: Novel triblock copolymer as a biocompatible and efficient gene delivery carrier. Biomacromolecules 2004. 5, 2487-2492.

77 J. S. Choi, K. NAM, J.-y. PARK, J.-B. Kim, J.-K. Lee, J.-s. PARK, Enhanced transfection efficiency of PAMAM dendrimer by surface modification with L-arginine. J. Controlled Release 2004. 99, 445-456.

78 J. H. Lee, Y.-b. Lim, J. S. Choi, Y. Lee, T.-i. Kim, H. J. Kim, J. K. Yoon, K. Kim, J.-s. PARK, Polyplexes assembled with internally quaternized PAMAM$\mathrm{OH}$ dendrimer and plasmid DNA have a neutral surface and gene delivery potency. Bioconjugate Chem. 2003. 14, 1214-1221.

79 M. Hussain, M. Shchepinov, M. Sohail, I. F. Benter, A. J. Hollins, E. M. Southern, S. Akhtar, A novel anionic dendrimer for improved cellular delivery of antisense oligonucleotides. J. Controlled Release 2004. 99, 139-155.

80 J. F. G. A. Jansen, E. W. Meijer, E. M. M. DE BRabander-VAN DEN BERG, The dendritic box: Shapeselective liberation of encapsulated guests. J. Am. Chem. Soc. 1995. 117, 4417-4418.

81 H. Ringsdorf, Structure and properties of pharmacologically active polymers. J. Polym. Sci. Polym. Symp. 1975. 51, 135-153.

82 H. Bader, H. Ringsdorf, B. ScHмIDT, Water-soluble polymers in medicine. Angew. Makromol. Chem. 1984. 123/124, 457-485.

83 J. Kopacek, Soluble biomedical polymers. Polym. Med. 1977. 7, 191-221.

84 E. R. Gillies, E. Dy, J. M. J. Fréchet, F. C. Szoka, Biological evaluation of polyester dendrimer: Poly(ethylene oxide) "bow-tie" hybrids with tunable molecular weight and architecture. Mol. Pharm. 2005. 2, 129-138.

85 P. Kolhe, J. Khandare, O. Pillai, S. Kannan, M. Lieh-Lai, R. M. Kannan, Preparation, cellular transport, and activity of polyamidoamine-based dendritic nanodevices with a high drug payload. Biomaterials 2006. 27, 660-669. 
86 S. C. Lee, R. Parthasarathy, K. Botwin, D. Kunneman, E. Rowold, G. Lange, J. Klover, A. AbegG, J. Zobel, T. Beck, T. Miller, W. Hood, J. Monahan, J. P. McKearn, R. Jansson, C. F. Voliva, Biochemical and immunological properties of cytokines conjugated to dendritic polymers. Biomed. Microdevices 2004. 6, 191-202.

87 F. Chaves, J. C. Calvo, C. Carvajal, Z. Rivera, L. Ramirez, M. Pinto, M. Trujillo, F. Guzman, M. E. Patarroyo, Synthesis, isolation and characterization of Plasmodium falciparum antigenic tetrabranched peptide dendrimers obtained by thiazolidine linkages. J. Pept. Res. 2001. 58, 307-316.

88 L. J. Cruz, E. Iglesias, J. C. Aguilar, L. J. GonzÁlez, O. Reyes, F. Albericio, D. Andreu, A comparative study of different presentation strategies for an HIV peptide immunogen. Bioconjugate Chem. 2004. $15,112-120$.

89 F. SÁnchez-Sancho, E. PérezInestrosa, R. Suau, C. Mayorga, M. J. Torres, M. Blanca, Dendrimers as carrier protein mimetics for IgE antibody recognition. Synthesis and characterization of densely penicilloylated dendrimers. Bioconjugate Chem. 2002. 13, 647-653.

90 S. Jaracz, J. Chen, L. V. Kuznetsova, I. Ojima, Recent advances in tumortargeting anticancer drug conjugates. Bioorg. Med. Chem. 2005. 13, 5043-5054.

91 Q. Cai, Y. Zhao, J. Bei, F. XI, S. WANG, Synthesis and properties of star-shaped polylactide attached to poly(amidoamine) dendrimer. Biomacromolecules 2003. 4, 828-834.

92 N. Malik, E. G. Evagorou, R. Duncan, Dendrimer-platinate: A novel approach to cancer chemotherapy. Anticancer Drugs 1999. 10, 767-776.

93 D. Shabat, R. J. Amir, A. Gopin, N. Pessah, M. Shamis, Chemical adaptor systems. Chem. Eur. J. 2004. 10, 2626-2634.

94 M. Shamis, H. N. Lode, D. Shabat, Bioactivation of self-immolative dendritic prodrugs by catalytic antibody 38C2. J. Am. Chem. Soc. 2004. 126, 1726-1731.

95 R. J. Amir, N. Pessah, M. Shamis, D. ShaBAT, Self-immolative dendrimers. Angew. Chem. Int. Ed. 2003. 42, 4494-4499.

96 F. M. H. de Groot, C. Albrecht, R. Koekroek, P. H. Beusker, H. W. Scheeren, "Cascade-release dendrimers" liberate all end groups upon a single triggering event in the dendritic core. Angew. Chem. Int. Ed. 2003. 42, 4940-4494.

97 A. T. Florence, T. Sakthivel, I. Тотн, Oral uptake and translocation of a polylysine dendrimer with a lipid surface. J. Controlled Release 2000. 65, 253-259.

98 R. Wiwattanapatapee, B. CarreñoGómez, N. Malik, R. Duncan, Anionic PAMAM dendrimers rapidly cross adult rat intestine in vitro: A potential oral delivery system? Pharm. Res. 2000. 17, 991-998.

99 A. T. Florence, N. Hussain, Transcytosis of nanoparticle and dendrimer delivery systems: Evolving vistas. Adv. Drug Delivery Rev. 2001. 50, S69-S89.

100 M. Ruponen, P. Honkakoski, S. Rönkкö, J. Pelkonen, M. Tammi, A. URTTI, Extracellular and intracellular barriers in non-viral gene delivery. J. Controlled Release 2003. 93, 213-217.

101 R. F. Barth, D. M. Adams, A. H. Soloway, F. Alam, M. V. Darby, Boronated starburst dendrimermonoclonal antibody immunoconjugates: Evaluation as a potential delivery system for neutron capture therapy. Bioconjugate Chem. 1994. 5 , 58-66.

102 S. Hong, A. U. Bielinska, A. Mecke, B. Keszler, J. L. Beals, X. Shi, L. Balogh, B. OrR, J. R. Baker JR, M. M. BANASZAK Holl, Interaction of poly(amidoamine) dendrimers with supported lipid bilayers and cells: Hole formation and the relation to transport. Bioconjugate Chem. 2004. 15, 774-782.

103 A. Mecke, S. Uppuluri, T. M. Sassanella, D.-K. Lee, A. 
RAMAmoorthy, J. R. BAKer JR, B. G. ORR, M. M. BANASZAK Holl, Direct observation of lipid bilayer disruption by poly(amidoamine) dendrimers. Chem. Phys. Lipids 2004. 132, 3-14.

104 G.-D. Zhang, A. Harada, N. Nishiyama, D.-L. Jiang, H. Koyam, T. Aida, K. KataOK, Polyion complex micelles entrapping cationic dendrimer porphyrin: Effective photosensitizer for photodynamic therapy of cancer. J. Controlled Release 2003. 93, 141-150.

105 J. C. Roberts, M. K. Bhalgat, R. T. Zera, Preliminary biological evaluation of polyamidoamine (PAMAM) Starburst ${ }^{\mathrm{TM}}$ dendrimers. J. Biomed. Mater. Res. 1996. 30, 53-65.

106 H. Kobayashi, S. Kawamoto, T. Saga, N. Sato, A. Hiraga, T. Ishimori, J. Konishi, K. Togashi, M. W. Brechbiel, Positive effects of polyethylene glycol conjugation to generation-4 polyamidoamine dendrimers as macromolecular MR contrast agents. Magn. Reson. Med. 2001. 46, 781-788.

107 N. RöCKEndoRF, T. K. LindHoRst, Glycodendrimers. Top. Curr. Chem. 2001. 217, 201-238.

108 L. Crespo, G. Sanclimens, M. Pons, E. Giralt, M. Roho, F. Albericio, Peptide and amide bond-containing dendrimers. Chem. Rev. 2005. 105, 1663-1681.

109 M. S. Shchepinov, I. A. Udalova, A. J. Brigman, E. M. Southern, Oligonucleotide dendrimers: Synthesis and use as polylabelled DNA probes. Nucleic Acids Res. 1997. 25, 4447-4454.

110 T. W. Nilsen, J. Grayzel, W. Prensky, Dendritic nucleic acid structures. J. Theoret. Biol. 1997. 187, 273-284.

111 R. H. E. Hudson, M. J. Damha, Nucleic acid dendrimers: Novel biopolymer structures. J. Am. Chem. Soc. 1998. 115, 2119-2124.

112 Y. Suzuki, T. Отомо, Н. OкAZI, Н. SAwaI, Synthesis and properties of a new type DNA dendrimer. Nucleic Acids Symp. Ser. 2000. 44, 125-126.

113 Y. Li, Y. D. Tseng, S. Y. Kwon, L. D’Espaux, J. S. Bunch, P. L.
McEuen, D. Luo, Controlled assembly of dendrimer-like DNA. Nat. Mater. 2004. 3, 38-42.

114 K. T. Al-Jamal, T. Sakthivel, A. T. Florence, Dendrisomes: Vesicular structures derived from a cationic lipidic dendron. J. Pharm. Sci. 2005. 94, 102-113.

115 S. Takeoka, K. Mori, H. Ohkawa, K. Sou, E. Tsuchida, Synthesis and assembly of poly(ethylene glycol)lipids with mono-, di-, and tetraacyl chains and a poly(ethylene glycol) chain of various molecular weights. J. Am. Chem. Soc. 2000. 122, 7927-7935.

116 H. Frey, R. HAaG, Dendritic polyglycerol: A new versatile biocompatible-material. J. Biotechnol. 2002. 90, 257-267.

117 M. F. Neerman, W. Zhang, A. R. Parrish, E. E. Simanek, In vitro and in vivo evaluation of a melamine dendrimer as a vehicle for drug delivery. Int. J. Pharm. 2004. 281, 129-132.

118 A.-M. Caminade, J.-P. Majoral, Nanomaterials based on phosphorus dendrimers. Acc. Chem. Res. 2004. 37, 341-348.

119 X.-Y. Wu, S.-W. Huang, J.-T. ZhanG, R.-X. ZHuo, Preparation and characterization of novel physically cross-linked hydrogels composed of poly(vinyl alcohol) and amineterminated polyamidoamine dendrimer. Macromol. Biosci. 2004. 4, 71-75.

120 H. R. Ihre, O. L. Padilla De Jesús, F. C. SZoKa JR, J. M. J. Fréchet, Polyester dendritic systems for drug delivery applications: Design, synthesis, and characterization. Bioconjugate Chem. 2002. 13, 443-452.

121 M. J. Cloninger, Biological applications of dendrimers. Curr. Opin. Chem. Biol. 2002. 6, 742-748.

122 L. S. Heuser, F. N. Miller, Differential macromolecular leakage from the vasculature of tumors. Cancer 1986. 57, 461-464.

123 H. Maeda, J. Wu, T. SAWA, Y. Matsumura, K. Hori, Tumor vascular permeability and the EPR effect in 
macromolecular therapeutics: A review. J. Controlled Release 2000. 65, 271-284.

124 Y. Matsumura, H. Maeda, A new concept for macromolecular therapeutics in cancer chemotherapy: Mechanism of tumoritropic accumulation of proteins and the antitumor agent smancs. Cancer Res. 1986. 46, 6387-6392.

125 L. W. Seymour, Passive tumor targeting of soluble macromolecules and drug conjugates. Crit. Rev. Ther. Drug Carrier Syst. 1992. 9, 135-187.

126 D. Liu, A. Mori, L. Huang, Role of liposome size and RES blockade in controlling biodistribution and tumor uptake of GM1-containing liposomes. Biochim. Biophys. Acta 1992. 1104, 95-101.

127 A. Nagayasu, T. Shimooka, Y. Kinouchi, K. Uchiyama, Y. TAKEICHI, H. KiWADA, Effects of fluidity and vesicle size on antitumor activity and myelosuppressive activity of liposomes loaded with daunorubicin. Biol. Pharm. Bull. 1994. 17, 935-939.

128 R. K. JaIN, Barriers to drug-delivery in solid tumors. Sci. Am. 1994. 271, 58-65.

129 Y. Tabata, Y. Murakami, Y. IKada, Tumor accumulation of poly(vinyl alcohol) of different sizes after intravenous injection. J. Controlled Release 1998. 50, 123-133.

130 S. Zalipsky, N. Mullah, J. A. Harding, J. Gittelman, L. Guo, S. A. DeFrees, Poly(ethylene glycol)-grafted liposomes with oligopeptide or oligosaccharide ligands appended to the termini of the polymer chains. Bioconjugate Chem. 1997. 8, 111-118.

131 J. A. Reddy, V. M. Allagadda, C. P. LEAMON, Targeting therapeutic and imaging agents to folate receptor positive tumors. Curr. Pharm. Biotechnol. 2005. 6, 131-150.

132 C. P. Leamon, J. A. Reddy, Folatetargeted chemotherapy. Adv. Drug Delivery Rev. 2004. 56, 1127-1141.

133 E. J. Roy, U. Gawlick, B. A. OrR, D. M. Kranz, Folate-mediated targeting of $\mathrm{T}$ cells to tumors.
Adv. Drug Delivery Rev. 2004. 56, 1219-1231.

134 Y. Choi, T. Thomas, A. Kotlyar, M. T. Islam, J. R. Baker, Synthesis and functional evaluation of DNAassembled polyamidoamine (PAMAM) dendrimer clusters with cancer cellspecific targeting. Chem. Biol. 2005. $12,35-43$.

135 D. LAHeru, E. M. JAFFeE, Immunotherapy for pancreatic cancer science driving clinical progress. Nat. Rev. Cancer 2005. 5, 549-467.

136 M. Harris, Monoclonal antibodies as therapeutic agents for cancer. Lancet Oncol. 2004. 5, 292-302.

137 M. Z. Lin, M. A. Teitell, G. J. SCHILler, The evolution of antibodies into versatile tumor-targeting agents. Clin. Cancer Res. 2005. 11, 129-138.

138 J.-Y. ZHANG, Tumor-associated antigen arrays to enhance antibody detection for cancer diagnosis. Cancer Detect. Prev. 2004. 28, 114-118.

139 R. E. Kontermann, Recombinant bispecific antibodies for cancer therapy. Acta Pharmacol. Sin. 2005. 26, 1-9.

140 P. A. Trail, D. H. King, G. M. Duвошснік, Monoclonal antibody drug immunoconjugates for targeted treatment of cancer. Cancer Immunol. Immunother. 2003. 52, 328-337.

141 G. C. McDonald, N. Glover, Effective tumor targeting: Strategies for the delivery of armed antibodies. Curr. Opin. Drug Discov. Devel. 2005. 8, 177-183.

142 S. V. Govindan, G. L. Griffiths, H. J. Hansen, I. D. Horak, D. M. Golden berG, Cancer therapy with radiolabeled and drug/toxinconjugated antibodies. Technol. Cancer Res. Treat. 2005. 4, 375-392.

143 D. J. FitzGerald, R. Kreitman, W. Wilson, D. Squires, I. Pastan, Recombinant immunotoxins for treating cancer. Int. J. Med. Microbiol. 2004. 293, 577-582.

144 K. Sandvig, S. Grimmer, T. G. Iversen, K. Rodal, M. L. Torgersen, P. Nicoziani, B. van Deurs, Ricin transport into cells: Studies of endocytosis and intracellular 
transport. Int. J. Med. Microbiol. 2000. 290, 415-420.

145 S. OlSNES, The history of ricin, abrin and related toxins. Toxicon 2004. 44, 361-370.

146 A. Gruaz-Guyon, O. Raguin, J. BARBET, Recent advances in pretargeted radioimmunotherapy. Curr. Med. Chem. 2005. 12, 319-338.

147 J. C. Roberts, Y. E. Adams, D. A. Tomalia, J. A. Mercer-Smith, D. K. LAVAlLEe, Using starburst dendrimers as linker molecules to radiolabel antibodies. Bioconjugate Chem. 1990. 1, 305-308.

148 P. Singh, Terminal groups in Starburst dendrimers: Activation and reactions with proteins. Bioconjugate Chem. 1998. 9, 54-63.

149 H. Kobayashi, N. Sato, T. Saga, Y. NaKamoto, T. Ishimori, S. Toyama, K. Togashi, J. Konishi, M. W. Brechbiel, Monoclonal antibodydendrimer conjugates enable radiolabeling of antibody with markedly high specific activity with minimal loss of immunoreactivity. Eur. J. Nucl. Med. Mol. Imaging 2000. 27, 1334-1339.

150 N. Fischer-Durand, M. Salmain, B. Rudolf, A. Vessières, J. Zakrzewski, G. JAOUEN, Synthesis of metalcarbonyl-dendrimer-antibody immunoconjugates: Towards a new format for carbonyl metallo immunoassay. ChemBioChem 2004. 5, 519525.

151 T. P. Thomas, A. K. Patri, A. Myc, M. T. Myaing, J. Y. Ye, T. B. Norris, J. R. BAKER JR, In vitro targeting of synthesized antibody-conjugated dendrimer nanoparticles. Biomacromolecules 2004. 5, 2269-2274.

152 A. K. Patri, A. Myc, J. Beals, T. P. Thomas, N. H. Bander, J. R. BAKer $J_{R}$, Synthesis and in vitro testing of J591 antibody-dendrimer conjugates for targeted prostate cancer therapy. Bioconjugate Chem. 2004. 15, 1174-1181.

153 S. S. Nigavekar, L. Y. Sung, M. Lianes, A. EL-Jawahri, T. S. Lawrence, C. W. Becker, L. Balogh, M. K. Khan, ${ }^{3} \mathrm{H}$ Dendrimer nano- particle organ/tumor distribution.

Pharm. Res. 2004. 21, 476-483.

154 H. Kobayashi, C. Wu, M.-K. Kim, C. H. Paik, J. A. Carrasquillo, M. W. BRECHBIEL, Evaluation of the in vivo biodistribution of indium-111 and yttrium-88 labeled dendrimer-1B4MDTPA and its conjugation with antiTac monoclonal antibody. Bioconjugate Chem. 1999. 10, 103-111.

155 M. Mamede, T. Saga, H. Kobayashi, T. Ishimori, T. Higashi, N. Sato, M. W. Brechbiel, J. Konishi, Radiolabeling of avidin with very high specific activity for internal radiation therapy of intraperitoneally disseminated tumors. Clin. Cancer Res. 2003. 9, 3756-3762.

156 D. S. Wilbur, P. M. Pathare, D. K. Hamlin, K. R. Buhler, R. L. Vessella, Biotin reagents for antibody pretargeting. 3. Synthesis, radioiodination, and evaluation of biotinylated starburst dendrimers. Bioconjugate Chem. 1998. 9, 813-825.

157 N. Malik, R. Wiwattanapatapee, R. Klopsch, K. Lorenz, H. Frey, J. W. Weener, E. W. Meijer, W. Paulus, R. Duncan, Dendrimers: Relationship between structure and biocompatibility in vitro, and preliminary studies on the biodistribution of ${ }^{125}$ I-labelled polyamidoamine dendrimers in vivo. J. Controlled Release 2000. 65, 133-148.

158 W. YANG, R. F. Barth, G. Wu, A. K. Bandyopadhyaya, B. T. S.

Thirumamagal, W. TJarks, P. J. Binns, K. J. Riley, H. Patel, J. A. Coderre, M. J. Ciesielski, R. A. FENSTERMAKER, Boronated epidermal growth factor as a delivery agent for neutron capture therapy of EGF receptor positive gliomas. Appl. Radiat. Isot. 2004. 61, 981-985.

159 H. Kobayashi, M. W. Brechbiel, Dendrimer-based macromolecular MRI contrast agents: Characteristics and application. Mol. Imaging 2003. 2, $1-10$.

160 S.-E. Stiriba, H. Frey, R. HaAg, Dendritic polymers in biomedical applications: From potential to clinical use in diagnostics and therapy. Angew. Chem. Int. Ed. 2002. 41, 1329-1334. 
161 T. Kaminaga, T. TAKeshita, I. KIMURA, Role of magnetic resonance imaging for evaluation of tumors in the cardiac region. Eur. Radiol. 2003. 13(Suppl 4), L1-L10.

162 P. de Graaf, F. Barkhof, A. C. Moll, S. M. IMHof, D. L. KNol, P. van DER Valk, J. A. Castelijns, Retinoblastoma: MR imaging parameters in detection of tumor extent. Radiology 2005. 235, 197-207.

163 M. D. Ogan, U. Schmied, M. E. Mosely, W. Grodd, H. PaAjanen, R. C. Brasch, Albumin labeled with Gd-DTPA. An intravascular contrastenhancing agent for magnetic resonance blood pool imaging: Preparation and characterization. Invest. Radiol. 1987. 22, 665-671.

164 L. D. Margerum, B. K. Campion, M. Koo, N. Shargill, J. Lai, A. Marumoto, P. C. Sontum, Gadolinium(III) DO31 macrocycles and polyethylene glycol coupled to dendrimers. Effect of molecular wieght on physical and biological properties of macromolecular magnetic resonance imaging agents. J. Alloys Compd. 1997. 249, 185-190.

165 S. D. Konda, S. Wang, M. BReCHBiel, E. C. Wiener, Biodistribution of a $153 \mathrm{Gd}$-folate dendrimer, generation $=4$, in mice with folate-receptor positive and negative ovarian tumor xenografts. Invest. Radiol. 2002. 37, 199-204.

166 H. Kobayashi, S. Kawamoto, S.-K. Jo, H. L. Bryant JR, M. W. Brechbiel, R. A. Star, Macromolecular MRI contrast agents with small dendrimers: Pharmacokinetic differences between sizes and cores. Bioconjugate Chem. 2003. 14, 388-394.

167 S. Guccione, K. C. Li, M. D. BEDNARSKI, Molecular imaging and therapy directed at the neovasculature in pathologies. How imaging can be incorporated into vascular-targeted delivery systems to generate active therapeutic agents. IEEE Eng. Med. Biol. Mag. 2004. 23, 50-60.

168 K. Onitsuka, M. Fujimoto, H. Kitajima, N. Ohshiro, F. Takei, S. TaKahashi, Convergent synthesis of platinum-acetylide dendrimers. Chem. Eur. J. 2004. 10, 6433-6446.

169 R. F. Barth, A. H. Soloway, Boron neutron capture therapy of primary and metastatic brain tumors. Mol. Chem. Neuropathol. 1994. 21, 139-154.

170 L. Liu, R. F. Barth, D. M. Adams, A. H. Soloway, R. A. Reisfeld, Bispecific antibodies as targeting agents for boron neutron capture therapy of brain tumors. J. Hematother. 1995. 4, 477-483.

171 F. Alam, A. H. Soloway, R. F. Barth, N. Mafune, D. M. Adams, W. H. КNотн, Boron neutron capture therapy: Linkage of a boronated macromolecule to monoclonal antibodies directed against tumorassociated antigens. J. Med. Chem. 1989. 32, 2326-2330.

172 R. F. Barth, G. Wu, W. Yang, P. J. Binns, K. J. Riley, H. Patel, J. A. Coderre, W. TJarks, A. K. Bandyopadhyaya, B. T. S. Thirumamagal, M. J. Ciesielski, R. A. Fenstermaker, Neutron capture therapy of epidermal growth factor $(+)$ gliomas using boronated cetuximab (IMC-C225) as a delivery agent. Appl. Radiat. Isot. 2004. 61, 899-903.

173 A. Quintana, E. Raczka, L. Piehler, I. Lee, A. Myc, I. Majoros, A. K. Patri, T. Thomas, J. Mulé, J. R. BAKER JR, Design and function of a dendrimer-based therapeutic nanodevice targeted to tumor cells through the folate receptor. Pharm. Res. 2002. 19, 1306-1310.

174 J. W. Dennis, S. Laferte, C. Waghorne, M. L. Breitman, R. S. Kerbel, ß1-6 branching of Asn-linked oligosaccharides is directly associated with metastasis. Science 1987. 236, 236-239.

175 S. SELL, Cancer-associated carbohydrates identified by monoclonal antibodies. Hum. Pathol. 1990. 21, 1003-1019.

176 A. Кobata, J. Amano, Altered glycosylation of proteins produced by malignant cells, and application for the diagnosis and immunotherapy of tumours. Immunol. Cell Biol. 2005. 83, 429-439. 
177 J. W. Dennis, M. Granovsky, C. E. WARren, Protein glycosylation in development and disease. BioEssays 1999. 21, 412-421.

178 L. Tong, G. Baskaran, M. B. Jones, J. K. Rhee, K. J. YARema, Glycosylation changes as markers for the diagnosis and treatment of human disease, in Biochemical and Genetic Engineering Reviews, S. HARDING (Ed.), 2003, Intercept Limited, Andover, Hampshire, UK. pp. 199-244.

179 J. R. Allen, C. R. Harris, S. J. Danishefsky, Pursuit of optimal carbohydrate-based anticancer vaccines: Preparation of a multiantigenic unimolecular glycopeptide containing the Tn, MBr1, and LewisY antigens. J. Am. Chem. Soc. 2001. 123, 1890-1897.

180 J. R. Allen, J. G. Allen, X. F. Zhang, L. J. Williams, A. Zatorski, G. Ragupathi, P. O. Livingston, S. J. DANISHEFSKY, A second generation synthesis of the MBr1 (Globo-H) breast tumor antigen: New application of the $N$-pentenyl glycoside method for achieving complex carbohydrate protein linkages. Chemistry 2000. 6, 1366-1375.

181 V. Kudryashov, P. W. Glunz, L. J. Williams, S. Hintermann, S. J. Danishefsky, K. O. Lloyd, Toward optimized carbohydrate-based anticancer vaccines: Epitope clustering, carrier structure, and adjuvant all influence antibody responses to LewisY conjugates in mice. Proc. Natl. Acad. Sci. U.S.A. 2001. 98, 3264-3269.

182 H. Kayser, R. Zeitler, C. Kannicht, D. Grunow, R. Nuck, W. Reutter, Biosynthesis of a nonphysiological sialic acid in different rat organs, using $N$-propanoyl-D-hexosamines as precursors. J. Biol. Chem. 1992. 267, 16934-16938.

183 K. J. Yarema, L. K. Mahal, R. E. Bruehl, E. C. Rodriguez, C. R. BERTOZZI, Metabolic delivery of ketone groups to sialic acid residues. Application to cell surface glycoform engineering. J. Biol. Chem. 1998. 273, 31168-31179.

184 L. K. Mahal, K. J. Yarema, C. R. Bertozzi, Engineering chemical reactivity on cell surfaces through oligosaccharide biosynthesis. Science 1997. 276, 1125-1128.

185 E. Saxon, C. R. Bertozzi, Cell surface engineering by a modified Staudinger reaction. Science 2000. 287, 2007-2010.

186 S.-G. SampathKumar, A. V. Li, M. B. Jones, Z. Sun, K. J. Yarema, Metabolic installation of thiols into sialic acid modulates adhesion and stem cell biology. Nat. Chem. Biol. 2006. 2, 149-152.

187 L. K. Mahal, K. J. Yarema, G. A Lemieux, C. R. Bertozzi, Chemical approaches to glycobiology: Engineering cell surface sialic acids for tumor targeting, in Sialobiology and Other Novel Forms of Glycosylation, Y. Inoue, Y. C. Lee, F. A. Troy II (Eds.), 1999, Gakushin Publishing Company, Osaka, Japan, pp. 273-280.

188 D. A. Nauman, C. R. Bertozzi, Kinetic parameters for small-molecule drug delivery by covalent cell surface targeting. Biochim. Biophys. Acta 2001. 1568, 147-154.

189 S.-G. Sampath Kumar, K. J. Yarema, Targeting cancer cells with dendrimers. Chem. Biol. 2005. 12, 5-6. 